\title{
AN EXISTENCE RESULT FOR ANISOTROPIC QUASILINEAR PROBLEMS
}

\author{
OSCAR AGUDELO AND PAVEL DRÁBEK
}

\begin{abstract}
We study existence of solutions for a boundary degenerate (or singular) quasilinear equation in a smooth bounded domain under Dirichlet boundary conditions. We consider a weighted $p$-Laplacian operator with a coefficient that is locally bounded inside the domain and satisfying certain additional integrability assumptions. Our main result applies for boundary value problems involving continuous non-linearities having no growth restriction, but provided the existence of a sub and a supersolution is guaranteed. As an application, we present an existence result for a boundary value problem with a non-linearity $f(u)$ satisfying $f(0) \leq 0$ and having $(p-1)$-sublinear growth at infinity.
\end{abstract}

Key words: $p$-Laplacian, quasilinear eigenvalue problems, subsolution and supersolution, weighted Sobolev spaces, Kato estimates, $(p-1)$-sublinearity.

MSC Classification: 35A01, 35J25, 35J60, 35J62, 35J70, 35J92.

\section{INTRODUCTION}

In this work we study the degenerate (or singular) boundary value problem $(B V P)$

$$
\left\{\begin{aligned}
-\operatorname{div}\left(a(x)|\nabla u|^{p-2} \nabla u\right) & =F(x, u) & & \text { in } \quad \Omega \\
u & =0 & & \text { on } \quad \partial \Omega,
\end{aligned}\right.
$$

where $N \geq 2, p \in(1, N)$ and $\Omega \subset \mathbb{R}^{N}$ is a smooth bounded domain.

In order to formulate our assumptions on the function $a: \Omega \rightarrow \mathbb{R}$, we consider first $\rho_{0}>0$ small so that in the set $\Omega_{\rho_{0}}:=\left\{x \in \Omega: 0<\operatorname{dist}(x, \partial \Omega)<\rho_{0}\right\}$ the distance function

$$
\Omega_{\rho_{0}} \ni x \mapsto \operatorname{dist}(x, \partial \Omega)
$$

satisfies that given any $x \in \Omega_{\rho_{0}}$, there exists a unique $z_{x} \in \partial \Omega \operatorname{such}$ that $\operatorname{dist}(x, \Omega)=$ $\operatorname{dist}\left(x, z_{x}\right)$ (see also subsection 2.3). We consider also a positive function a : $\left(0, \rho_{0}\right) \rightarrow(0, \infty)$ and a fixed positive number $s$ such that

$$
\mathrm{a} \in L^{1}\left(0, \rho_{0}\right) \cap L_{l o c}^{\infty}\left(0, \rho_{0}\right), \quad \mathrm{a}^{-1} \in L^{s}\left(0, \rho_{0}\right) \quad \text { and } \quad s>\max \left(\frac{N}{p}, \frac{1}{p-1}\right) .
$$

In particular, $\mathrm{a}^{-\frac{1}{p-1}} \in L^{1}\left(0, \rho_{0}\right)$.

The assumptions for the function $a$ are the following:

(A1) $a \in L_{l o c}^{\infty}(\Omega)$ and for any open set $D$ with $\bar{D} \subset \Omega, \inf _{D} a>0$; 
(A2) for every $x \in \Omega_{\rho_{0}}$,

$$
a(x)=\mathrm{a}(\operatorname{dist}(x, \partial \Omega)) .
$$

Assumptions (A1) and (A2) imply that the differential operator in (1.1) is uniformly elliptic on any subdomain of $\Omega$, but not necessarily near the boundary of the domain.

These hypotheses also yield that $a \in L^{1}(\Omega)$ and $a^{-1} \in L^{s}(\Omega)$ (see Section 2.1 ), which will allow us to put the equation (1.1) into an appropriate functional analytic setting.

To formulate the assumptions on the function $F: \Omega \times \mathbb{R} \rightarrow \mathbb{R}$, we first let $s>0$ be as in (1.3) and denote

$$
p_{s}:=\frac{p s}{s+1}, \quad p_{s}^{*}:=\frac{N p_{s}}{N-p_{s}}=\frac{N p s}{N(s+1)-p s} .
$$

Using that $s>\frac{N}{p}>1$, we find that

$$
p_{s}<p<p_{s}^{*}<\frac{N p}{N-p}
$$

Next, fix $q \in\left[p, p_{s}^{*}\right)$ and set $\frac{q}{q-p}:=\infty$, if $q=p$. Consider also a measurable function $b$ satisfying the following:

(B1) $b \in L^{\frac{q}{q-p}}(\Omega)$;

(B2) there exist constants $0<c_{1}<c_{2}$ such that

$$
c_{1} \leq \liminf _{\operatorname{dist}(x, \partial \Omega) \rightarrow 0} a^{\frac{1}{p-1}}(x) b(x) \leq \limsup _{\operatorname{dist}(x, \partial \Omega) \rightarrow 0} a^{\frac{1}{p-1}}(x) b(x) \leq c_{2} .
$$

In particular, $b \neq 0$ and $b \geq 0$ a.e. in $\Omega$.

As for the function $F: \Omega \times \mathbb{R} \rightarrow \mathbb{R}$, we assume it is a Caratheodory function satisfying the growth condition:

for every $M>0$, there exists $C_{M}>0$ such that for every $\zeta \in \mathbb{R}$ with $|\zeta| \leq M$ and for a.e. $x \in \Omega$,

$$
|F(x, \zeta)| \leq C_{M} b(x) .
$$

We use weighted Sobolev spaces to study the BVP (1.1). The Sobolev space $W^{1, p}(\Omega, a)$ is defined as the class of functions $v \in W_{l o c}^{1, p}(\Omega)$ such that

$$
\|v\|_{W^{1, p}(\Omega, a)}:=\left(\int_{\Omega}|v|^{p} d x+\int_{\Omega} a(x)|\nabla v|^{p} d x\right)^{\frac{1}{p}}<\infty .
$$

The Sobolev space $W_{0}^{1, p}(\Omega, a)$ is defined as the closure of $C_{c}^{\infty}(\Omega)$ with respect to the norm $\|\cdot\|_{W^{1, p}(\Omega, a)} \cdot$

A function $u \in W_{0}^{1, p}(\Omega, a)$ is called a solution of (1.1) if $F(\cdot, u(\cdot)) \in L^{\frac{p}{p-1}}(\Omega)$ and

$$
\forall \varphi \in W_{0}^{1, p}(\Omega, a): \quad \int_{\Omega} a(x)|\nabla u|^{p-2} \nabla u \cdot \nabla \varphi d x=\int_{\Omega} F(x, u) \varphi d x .
$$


A function $u \in W^{1, p}(\Omega, a) \cap C(\bar{\Omega})$ is called a subsolution (supersolution respectively) of (1.1) if $F(\cdot, u(\cdot)) \in L^{\frac{p}{p-1}}(\Omega)$, for every $\varphi \in W_{0}^{1, p}(\Omega, a)$ with $\varphi \geq 0$ a.e. in $\Omega$,

$$
\int_{\Omega} a(x)|\nabla u|^{p-2} \nabla u \cdot \nabla \varphi d x \leq(\geq) \int_{\Omega} F(x, u) \varphi d x
$$

and $\max _{x \in \partial \Omega} u(x) \leq 0 \quad\left(\min _{x \in \partial \Omega} u(x) \geq 0\right)$.

Our first main result reads as follows.

Theorem 1.1. Let (A1)-(A2) and (B1)-(B2) hold and let F satisfy (1.6). Assume that $\underline{u}$ is a subsolution and $\bar{U}$ is a supersolution to (1.1) such that $\underline{u} \leq \bar{U}$ in $\Omega$. Then the $B V P$ (1.1) has a solution $u \in C(\bar{\Omega})$ such that $\underline{u} \leq u \leq \bar{U}$ in $\Omega$.

The proof of Theorem 1.1 is motivated by the techniques described in Chapter 5 in [10]. As an application of Theorem 1.1, we present existence of a positive solution for the BVP

$$
\left\{\begin{aligned}
-\operatorname{div}\left(a(x)|\nabla u|^{p-2} \nabla u\right) & =\lambda b(x) f(u) & & \text { in } \quad \Omega \\
u & =0 & & \text { on } \quad \partial \Omega,
\end{aligned}\right.
$$

where $\lambda>0$ is a positive parameter and $f:[0, \infty) \rightarrow \mathbb{R}$ is a continuous function satisfying the following hypotheses:

(f1) $f$ is $(p-1)$-sublinear, i.e., $\limsup _{\zeta \rightarrow \infty} \frac{f(\zeta)}{\zeta^{p-1}}=0$;

(f2) there exists $r \in(0, p-1)$ such that $\liminf _{\zeta \rightarrow \infty} \frac{f(\zeta)}{\zeta^{r}} \in(0, \infty]$.

(f3) $f(0) \leq 0$ and $\min _{0 \leq \zeta<\infty} f(\zeta)<0$.

Problem (1.9) finds its applications, for instance, in resource management models, see [5], and design of suspension bridges, see [13].

We present the function

$$
f(\zeta)=-A+\frac{\zeta^{p-1}}{\log (2+\zeta)} \quad \text { for } \quad \zeta \geq 0,
$$

where $A \geq 0$, as an example of a nonlinearity satisfying conditions (f1), (f2) and (f3).

Condition $f(0) \leq 0$ in (f3) implies that $u=0$ is a supersolution to (1.9). Under this condition, finding a positive subsolution for (1.9) becomes a subtle matter. We refer the reader to [2, 3, 4, 16, 19] and references therein, for results concerned with related boundary value problems in the uniformly elliptic case.

The authors in [1] showed the existence of a positive solution of equation (1.9) either in a ball or in $\mathbb{R}^{N}$, under a similar set of assumptions on $a, b$ and $f$, but assuming in addition 
the global radial symmetry of the coefficient $a$ and instead of (f3), assuming that there exist $K_{0}, \delta>0$ and $\gamma \in(0,1)$ such that

$$
\text { for every } \quad \zeta \in(0, \delta), \quad-\frac{K_{0}}{\zeta^{\gamma}} \leq f(\zeta) \leq 0 .
$$

As remarked in [1], our hypotheses do not allow, in general, to obtain either $C_{l o c}^{1}$-regularity of weak solutions to (1.9), see [23], or Hopf's boundary lemma, see [24]. Instead, regularity of solutions relies on the local Hölder continuity due to Serrin, see [21]. In particular, global a priori $C^{1}$-estimates and therefore the construction of a subsolution of (1.9) is a much more challenging task. In this work, tools from differential geometry help us overcome this difficulty.

More precisely, we say that $\partial \Omega$ has non-negative mean curvature if for every $x \in \partial \Omega$,

$$
\sum_{i=1}^{N-1} k_{i}(x) \geq 0,
$$

where $k_{1}, \ldots, k_{N-1}$ are the principal curvatures of $\partial \Omega$.

This geometric notion generalizes convexity and it is equivalent to the monotonicity of the surface area element of $\partial \Omega$, see [14]. This monotonicity states that the $(N-1)$-dimensional volume of every subdomain $\omega \subset \partial \Omega$ does not decrease as $\omega$ approaches $\partial \Omega$.

From the PDE point of view, condition (1.10) implies that the distance function dist $(\cdot, \partial \Omega)$ is subharmonic in any subdomain of $\Omega_{\rho_{0}}$ (see (1.2)) and in essence this fact will allow us to construct an appropriate subsolution to (1.9) .

Our second main result reads as follows.

Theorem 1.2. Let $\Omega$ be a smooth bounded domain such that $\partial \Omega$ has non-negative mean curvature. Let (A1)-(A2) and (B1)-(B2) hold and let $f \in C[0, \infty)$ satisfy (f1)-(f3). Then, there exists $\lambda_{0}>0$ such that for every $\lambda \geq \lambda_{0}$ the $B V P(1.9)$ has a solution $u \in C(\bar{\Omega})$ which is positive in $\Omega$.

We refer the reader to [8, 18] for similar results in the uniformly elliptic setting.

In the case $a(x) \equiv 1$ and $p=2$, under slightly more restrictive assumptions on $f$ and with no assumptions on the geometry of $\partial \Omega$, the authors in [6] proved uniqueness of positive solutions of (1.9) for $\lambda>0$ large. We also refer the reader to [17] for non-existence of positive radial solutions of a related BVP in the superlinear setting.

We have considered assumption (A2) for the sake of clarity in our presentation. Although, we remark that Theorems 1.1 and 1.2 are still valid if instead of (A2), we assume the existence of functions $\mathrm{a}_{1}, \mathrm{a}_{2}:\left(0, \rho_{0}\right) \rightarrow(0, \infty)$, both satisfying (1.3) and such that

$$
\mathrm{a}_{1}(\operatorname{dist}(x, \partial \Omega)) \leq a(x) \leq \mathrm{a}_{2}(\operatorname{dist}(x, \partial \Omega))
$$

for every $x \in \Omega_{\rho_{0}}$. The proofs require only straight forward changes and details are left to the reader. 
The paper is organized as follows. In Section 2 we present the preliminary results and a priori estimates needed for the proof of Theorem 1.1. The proof of Theorem 1.1 is presented in Section 3. In Section 4 we provide the proof Theorem 1.2 .

\section{PRELIMINARIES}

2.1. Weighted Sobolev spaces. Let $p \in(1, N), \Omega \subset \mathbb{R}^{N}$ be a smooth bounded domain and let $a: \Omega \rightarrow \mathbb{R}$ satisfy (A1) and (A2).

From these hypotheses and with the help of Fermi coordinates, we verify in subsection 2.3 that $a \in L^{1}(\Omega)$ and $a^{-1} \in L^{s}(\Omega)$. Observe also that the Hölder inequality and the fact that $s>\frac{1}{p-1}$ yield $a^{-\frac{1}{p-1}} \in L^{1}(\Omega)$.

Thus, the spaces $W^{1, p}(\Omega, a)$ and $W_{0}^{1, p}(\Omega, a)$, defined in Section 1 , are separable. They are also uniformly convex and therefore reflexive Banach spaces (see Chapter 1 in [9]).

We also notice from $(A 1)$ that for any $D$ with $\bar{D} \subset \Omega$,

$$
W^{1, p}(D, a)=W^{1, p}(D) \quad \text { and } \quad W_{0}^{1, p}(D, a)=W_{0}^{1, p}(D) .
$$

In what follows $\mathbb{1}_{E}$ denotes the characteristic function of a set $E \subset \mathbb{R}^{N}$. Also, for any $t \in \mathbb{R}$, write

$$
t^{+}:=\frac{t+|t|}{2}, \quad t^{-}:=\frac{t-|t|}{2},
$$

so that $t^{+}=\max (t, 0)$ and $t^{-}=\min (t, 0)$.

Our first lemma concerns with algebraic properties of the spaces $W^{1, p}(\Omega, a)$ and $W_{0}^{1, p}(\Omega, a)$.

Lemma 2.1. Under the hypotheses (A1) and (A2),

(i) if $v \in W^{1, p}(\Omega, a)$, then $|v|, v^{+}, v^{-}$belong to $W^{1, p}(\Omega, a)$ and

$$
\nabla v^{+}=\mathbb{1}_{\{v>0\}} \nabla v, \quad \nabla v^{-}=\mathbb{1}_{\{v<0\}} \nabla v
$$

(ii) if $v \in W_{0}^{1, p}(\Omega, a)$, then $|v|, v^{+}, v^{-} \in W_{0}^{1, p}(\Omega, a)$ and

(iii) if $v, w \in W^{1, p}(\Omega, a)$, then $\min (v, w), \max (v, w) \in W^{1, p}(\Omega, a)$.

Proof. (i) From (A1) and Theorem 7.8 in [15], $|v|, v^{+}, v^{-} \in W_{l o c}^{1, p}(\Omega)$ and (2.2) holds. Directly from (2.1) and (2.2), it follows that $|v|, v^{+}, v^{-} \in W^{1, p}(\Omega, a)$.

(ii) Let $v \in W_{0}^{1, p}(\Omega, a)$. There exists $\left\{v_{n}\right\}_{n} \subset C_{c}^{\infty}(\Omega)$ such that $v_{n} \rightarrow v$ strongly in $W_{0}^{1, p}(\Omega, a)$. Let $\left\{\varepsilon_{n}\right\}_{n} \subset(0, \infty)$ be such that $\varepsilon_{n} \rightarrow 0$ as $n \rightarrow \infty$ and set

$$
w_{n}:=\sqrt{v_{n}^{2}+\varepsilon_{n}^{2}}-\varepsilon_{n} \quad \text { for } \quad n \in \mathbb{N} .
$$

Observe that $w_{n} \in C_{c}^{\infty}(\Omega)$ since $\operatorname{supp} w_{n}=\operatorname{supp} v_{n}$. Also, it is direct to verify that $w_{n} \rightarrow|v|$ strongly in $W^{1, p}(\Omega, a)$ as $n \rightarrow \infty$. Therefore, $|v| \in W_{0}^{1, p}(\Omega, a)$ and from (2.1), $v^{+}, v^{-} \in W_{0}^{1, p}(\Omega, a)$.

(iii) Let $v, w \in W^{1, p}(\Omega, a)$. The proof is a direct consequence of (i) and the fact that

$$
\min (v, w)=(v-w)^{-}+w \text { and } \max (v, w)=(w-v)^{+}+v
$$


Lemma 2.2. Assume (A1) and (A2). Then, $W_{0}^{1, p}(\Omega, a)$ is the closure of the subspace

$$
X:=W^{1, p}(\Omega, a) \cap\left\{v \in C(\bar{\Omega}):\left.v\right|_{\partial \Omega}=0\right\}
$$

with respect to the norm $\|\cdot\|_{W^{1, p}(\Omega, a)}$.

Proof. Observe that $C_{c}^{\infty}(\Omega) \subset X$ and hence $W_{0}^{1, p}(\Omega, a)$ is contained in the closure of $X$. To prove the reverse inclusion, we claim first that $X$ is the closure of $W^{1, p}(\Omega, a) \cap C_{c}(\Omega)$ with respect to the norm $\|\cdot\|_{W^{1, p}(\Omega, a)}$.

To prove the claim we follow the lines of the proof of Theorem 2 from section 5.5 in [11]. This is done in two steps.

Step 1. Let $u \in X$ and let $x_{*} \in \partial \Omega$ be arbitrary, but fixed. With no loss of generality, assume that $x_{*}=\left(x_{*}^{\prime}, 0\right)$ with $x_{*}^{\prime} \in \mathbb{R}^{N-1}$. For any $\rho \in\left(0, \rho_{0}\right)$ (see (1.2)), denote

$$
\mathcal{C}_{\rho}\left(x_{*}\right):=D_{\rho}^{N-1}\left(x_{*}^{\prime}\right) \times(-\rho, \rho),
$$

where $D_{\rho}^{N-1}\left(x_{*}^{\prime}\right)$ is the ball in $\mathbb{R}^{N-1}$ of radius $\rho$ and centered at $x_{*}^{\prime}$.

After a local flattening procedure and relabelling of the axis, we may also assume that there exists $\rho_{*} \in\left(0, \rho_{0}\right)$ such that

$$
\begin{aligned}
\Gamma & :=\partial \Omega \cap \mathcal{C}_{\rho_{*}}\left(x_{*}\right)=\left\{\left(x^{\prime}, x_{N}\right): x_{N}=0\right\} \cap \mathcal{C}_{\rho_{*}}\left(x_{*}\right), \\
\Omega_{*} & :=\Omega \cap \mathcal{C}_{\rho_{*}}\left(x_{*}\right)=\left\{\left(x^{\prime}, x_{N}\right): x_{N}>0\right\} \cap C_{\rho_{*}}\left(x_{*}\right) .
\end{aligned}
$$

Let $\varepsilon>0$ be arbitrary, but fixed. Since $u \in W^{1, p}(\Omega, a)$, there exists $m_{*} \in \mathbb{N}$ with $m_{*} \geq \frac{1}{2 \rho_{*}}$ such that

$$
\forall m \geq m_{*}: \int_{\Gamma} \int_{0}^{\frac{2}{m}} a(x)|\nabla u|^{p} d x<\varepsilon .
$$

From (1.3) we may also assume that for any $m \geq m_{*}$,

$$
\left(\int_{\frac{1}{m}}^{\frac{2}{m}} \mathrm{a}(\zeta) d \zeta\right)\left(\int_{0}^{\frac{2}{m}} \mathrm{a}^{-\frac{1}{p-1}}(\zeta) d \zeta\right)^{p-1}<\varepsilon
$$

Let $\chi: \mathbb{R} \rightarrow[0,1]$ be a smooth cut-off function with $\chi \equiv 0$ in $(-\infty, 1]$ and $\chi \equiv 1$ in $[2, \infty)$.

For any $m \in \mathbb{N}$ with $m \geq m_{*}$, set

$$
\begin{aligned}
& \chi_{m}\left(x_{N}\right):=\chi\left(m x_{N}\right) \quad \text { for } 0<x_{N}<\infty \text {, } \\
& u_{m}(x):=\chi_{m}\left(x_{N}\right) u(x) \quad \text { for } \quad x \in \bar{\Omega}_{*} .
\end{aligned}
$$

Observe that $u_{m} \in W^{1, p}\left(\Omega_{*}, a\right) \cap C\left(\bar{\Omega}_{*}\right), u_{m}=0$ in $\Gamma \times\left(0, \frac{1}{m}\right)$ and

$$
\nabla u_{m}=\chi\left(m x_{N}\right) \nabla u+m \chi^{\prime}\left(m x_{N}\right) u \quad \text { a.e. in } \quad \Omega_{*} \text {. }
$$


Thus, there exists a constant $C>0$, depending only on $p$, such that for any $m \geq m *$ and a.e. in $\Omega_{*}$,

$$
\left|\nabla\left(u_{m}-u\right)\right|^{p} \leq C\left(1-\chi\left(m x_{N}\right)\right)^{p}|\nabla u|^{p}+C m^{p}\left|\chi^{\prime}\left(m x_{N}\right)\right|^{p}|u|^{p} .
$$

Denote $V_{*}:=\Omega \cap \mathcal{C}_{\frac{\rho *}{2}}\left(x_{*}\right)$. Taking $C$ larger if necessary, but still depending only on $p$,

$$
\begin{aligned}
& \int_{V_{*}} a(x)\left|\nabla\left(u_{m}-u\right)\right|^{p} d x \leq \underbrace{C \int_{\Gamma} \int_{0}^{\frac{2}{m}} a(x)|\nabla u|^{p} d x}_{I_{m}} \\
&+\underbrace{C m^{p} \int_{\Gamma} \int_{\frac{1}{m}}^{\frac{2}{m}} a\left(x^{\prime}, x_{N}\right)\left|u\left(x^{\prime}, x_{N}\right)\right|^{p} d x_{N} d x^{\prime}}_{I I_{m}} .
\end{aligned}
$$

From (2.3), for every $m \geq m_{*}$,

$$
0 \leq I_{m}<C \varepsilon
$$

Next, we estimate $I I_{m}$. Let $\left(x^{\prime}, x_{N}\right) \in \Gamma \times\left(\frac{1}{m}, \frac{2}{m}\right)$. Using Theorem 2 from section 4.9 .2 in [12], we estimate,

$$
\begin{aligned}
\left|u\left(x^{\prime}, x_{N}\right)\right| & \leq \underbrace{\left|u\left(x^{\prime}, 0\right)\right|}_{=0}+\int_{0}^{x_{N}} x_{N}\left|\nabla u\left(x^{\prime}, \zeta\right)\right| d \zeta \\
& \leq x_{N}\left(\int_{0}^{x_{N}} a^{-\frac{1}{p-1}}\left(x^{\prime}, \zeta\right) d \zeta\right)^{\frac{p-1}{p}}\left(\int_{0}^{x_{N}} a\left(x^{\prime}, \zeta\right)\left|\nabla u\left(x^{\prime}, \zeta\right)\right|^{p} d \zeta\right)^{\frac{1}{p}} .
\end{aligned}
$$

From (A2) and (2.7),

$$
\begin{aligned}
I I_{m} & \leq C m^{p} \int_{\Gamma} \int_{\frac{1}{m}}^{\frac{2}{m}} \mathrm{a}\left(x_{N}\right) x_{N}^{p}\left(\int_{0}^{x_{N}} \mathrm{a}^{-\frac{1}{p-1}}(\zeta) d \zeta\right)^{\frac{1}{p-1}}\left(\int_{0}^{x_{N}} a\left(x^{\prime}, \zeta\right)\left|\nabla u\left(x^{\prime}, \zeta\right)\right|^{p} d \zeta\right) d x_{N} d x^{\prime} \\
& \leq C\left(\int_{\frac{1}{m}}^{\frac{2}{m}} \mathrm{a}\left(x_{N}\right) d x_{N}\right)\left(\int_{0}^{\frac{2}{m}} \mathrm{a}^{-\frac{1}{p-1}}(\zeta) d \zeta\right)^{\frac{1}{p-1}} \int_{\Gamma} \int_{0}^{\frac{2}{m}} a(x)|\nabla u|^{p} d x,
\end{aligned}
$$

where $C>0$ is taken again larger if necessary, but still depending only on $p$.

Putting together (2.3), (2.4) and the latter inequalities,

$$
\begin{aligned}
I I_{m} & \leq C\left(\int_{\frac{1}{m}}^{\frac{2}{m}} \mathrm{a}\left(x_{N}\right) d x_{N}\right)\left(\int_{0}^{\frac{2}{m}} \mathrm{a}^{-\frac{1}{p-1}}(\zeta) d \zeta\right)^{\frac{1}{p-1}} I_{m} \\
& \leq C \varepsilon^{2} .
\end{aligned}
$$

Expressions (2.5), (2.6) and (2.8) yield

$$
\forall m \geq m_{*}: \int_{V_{*}} a(x)\left|\nabla\left(u_{m}-u\right)\right|^{p} d x \leq C(1+\varepsilon) \varepsilon .
$$


Since $u_{m} \rightarrow u$ in $L^{\infty}\left(V_{*}\right)$, we assume also that

$$
\forall m \geq m_{*}:\left\|u_{m}-u\right\|_{L^{p}\left(V_{*}\right)} \leq \varepsilon
$$

and therefore, for some $\tilde{C}>0$ independent of $\varepsilon$, and for every $m \geq m_{*}$,

$$
\left\|u_{m}-u\right\|_{W^{1, p}\left(V_{*}, a\right)} \leq \tilde{C} \varepsilon .
$$

Step 2. Let $v \in X$ and consider $\left\{V_{i}\right\}_{i=0}^{n}$ an open covering for $\bar{\Omega}$, where for $i=1, \ldots, m$, $V_{i}$ is a neigborhood of a point $x_{i} \in \partial \Omega$ as described in Step 1 and $\bar{V}_{0} \subset \Omega$.

Let $\varepsilon>0$ be arbitrary, but fixed. From Step 1, there exists $\tilde{\rho} \in\left(0, \rho_{0}\right)$ and for any $i=1, \ldots, m$, there exists $v_{i} \in W^{1, p}\left(V_{i}, a\right) \cap C\left(\overline{V_{i}}\right)$ such that

$$
\left\|v_{i}-v\right\|_{W^{1, p}\left(V_{i}, a\right)} \leq \varepsilon
$$

and $v_{i}=0$ in $V_{i} \cap \Omega_{\tilde{\rho}}$, where

$$
\Omega_{\tilde{\rho}}:=\{x \in \Omega: 0<\operatorname{dist}(x, \partial \Omega)<\tilde{\rho}\} .
$$

Since $W^{1, p}\left(V_{0}, a\right)=W^{1, p}\left(V_{0}\right)$, Theorem 1 from Section 5.3.1 in 11] yields the existence of $v_{0} \in C^{\infty}\left(\bar{V}_{0}\right)$ such that

$$
\left\|v_{0}-v\right\|_{W^{1, p}\left(V_{0}, a\right)} \leq \varepsilon
$$

Let $\left\{\chi_{i}\right\}_{i=0}^{m}$ be a smooth partition of the unity associated to the covering $\left\{V_{i}\right\}_{i=0}^{m}$. Set $\mathrm{v}_{i}:=\chi_{i} v_{i}$ and $v^{*}:=\sum_{i=0}^{m} \mathrm{v}_{i}$. Observe that $v^{*} \in W^{1, p}(\Omega, a) \cap C_{c}(\bar{\Omega})$.

We estimate

$$
\begin{aligned}
\left\|v^{*}-v\right\|_{W^{1, p}(\Omega, a)} & \leq \sum_{i=0}^{m}\left\|\mathrm{v}_{i}-\chi_{i} v\right\|_{W^{1, p}\left(V_{i}, a\right)} \\
& \leq \hat{C} \sum_{i=0}^{m}\left\|v_{i}-v\right\|_{W^{1, p}\left(V_{i}, a\right)} \\
& \leq \hat{C}(m+1) \varepsilon
\end{aligned}
$$

for some constant $\hat{C}>0$ depending only on $p$ and $\Omega$.

Since $\varepsilon>0$ and $v \in X$ are arbitrary, we conclude that $X$ is the closure of $W^{1, p}(\Omega, a) \cap$ $C_{c}(\Omega)$ with respect to the norm $\|\cdot\|_{W^{1, p}(\Omega, a)}$ which proves the claim.

We finish the proof now. Let $u \in X$ and let $\varepsilon>0$. From Steps 1 and 2 , there exists $v \in W^{1, p}(\Omega, a) \cap C_{c}(\Omega)$ such that

$$
\|u-v\|_{W^{1, p}(\Omega, a)} \leq \varepsilon
$$

Let $D$ be an open set such that $\operatorname{supp} v \subset D \subset \bar{D} \subset \Omega$. From $(A 1), W_{0}^{1, p}(D, a)=W_{0}^{1, p}(D)$ and for every $w \in W_{0}^{1, p}(D, a)$,

$$
\left(\inf _{D} a\right)^{\frac{1}{p}}\|w\|_{W_{0}^{1, p}(D)} \leq\|w\|_{W_{0}^{1, p}(D, a)} \leq\|a\|_{L^{\infty}(D)}^{\frac{1}{p}}\|w\|_{W_{0}^{1, p}(D)} .
$$


On the other hand, since $v \in W_{0}^{1, p}(D)$, there exists $w \in C_{c}^{\infty}(D)$ such that

$$
\|v-w\|_{W_{0}^{1, p}(D)} \leq\|a\|_{L^{\infty}(D)}^{-\frac{1}{p}} \varepsilon
$$

Therefore,

$$
\begin{aligned}
\|u-w\|_{W_{0}^{1, p}(\Omega, a)} & \leq\|u-v\|_{W_{0}^{1, p}(\Omega, a)}+\|v-w\|_{W_{0}^{1, p}(\Omega, a)} \\
& \leq \varepsilon+\|v-w\|_{W_{0}^{1, p}(D, a)} \\
& \leq \varepsilon+\|a\|_{L^{\infty}(D)}^{\frac{1}{p}}\|v-w\|_{W_{0}^{1, p}(D)} \\
& \leq 2 \varepsilon .
\end{aligned}
$$

Since $\varepsilon>0$ and $u \in X$ are arbitrary, we conclude that $X \subset W_{0}^{1, p}(\Omega, a)$. This completes the proof of Lemma 2.2 .

We complete this subsection with some remarks on Sobolev embeddings for the space $W^{1, p}(\Omega, a)$. From (1.4) and Hölder's inequality, the embedding

$$
W^{1, p}(\Omega, a) \hookrightarrow W^{1, p_{s}}(\Omega)
$$

is continuous (see [9]). Since $1<p_{s}<N$, from the standard Sobolev embeddings,

$$
W^{1, p}(\Omega, a) \hookrightarrow L^{m}(\Omega)
$$

with

$$
1 \leq m \begin{cases}\leq p_{s}^{*}, & \text { continuous embedding } \\ <p_{s}^{*}, & \text { compact embedding. }\end{cases}
$$

In particular, there exists $\mu_{0}>0$ such that for every $v \in W^{1, p}(\Omega, a)$,

$$
\mu_{0}\|v\|_{L^{p_{s}^{*}(\Omega)}} \leq\|v\|_{W^{1, p}(\Omega, a)} .
$$

From (1.3) $p_{s}^{*}>p$, which together with (2.9), yield

$$
W_{0}^{1, p}(\Omega, a) \hookrightarrow L^{p}(\Omega)
$$

Friedrich's inequality implies that the norm

$$
\|u\|_{W_{0}^{1, p}(\Omega, a)}:=\left(\int_{\Omega} a(x)|\nabla u|^{p} d x\right)^{\frac{1}{p}}
$$

in $W_{0}^{1, p}(\Omega, a)$ is equivalent to the norm $\|\cdot\|_{W^{1, p}(\Omega, a)}$. 
2.2. A priori estimates and regularity. Our next results concern with a priori estimates for solutions of (1.1).

Proposition 2.1. let (A1),(A2) and (B1) hold. Let $F(x, \zeta)$ be a Caratheodory function such that,

$$
|F(x, \zeta)| \leq b(x) \quad \text { for a.e. } \quad x \in \Omega, \quad \zeta \in \mathbb{R} .
$$

There exists a constant $C>0$ such that for every solution $u \in W_{0}^{1, p}(\Omega, a)$ of (1.1),

$$
\|u\|_{L^{\infty}(\Omega)} \leq C\left(1+\|b\|_{L^{1}(\Omega)}+\|u\|_{L^{p}(\Omega)}\right) .
$$

The proof of Proposition 2.1 follows the lines of Theorem 3.2 in [9] using Moser's iteration technique. We refer the reader also to Appendix B in [22] and references therein for the case $p=2$.

Proof. Consider first the case $p<q$. let $u \in W_{0}^{1, p}(\Omega, a)$ be a solution of (1.1) and write $u=u^{+}+u^{-}$.

For $\varepsilon>0$, denote

$$
C(\varepsilon):=\frac{(1+\varepsilon)^{p}}{1+\varepsilon p}
$$

Let $M, \varepsilon>0$ be arbitrary and consider the function

$$
v_{M, \varepsilon}(x):=\min \left(|u|^{1+\varepsilon}, M\right), \quad x \in \Omega .
$$

From part (iii) in Lemma 2.1, $v_{M, \varepsilon} \in W^{1, p}(\Omega, a)$ and observe that

$$
\begin{aligned}
\int_{\Omega} a(x)\left|\nabla v_{M, \varepsilon}\right|^{p} d x & =\int_{\left\{|u|^{1+\varepsilon} \leq M\right\}} a(x)\left|\nabla\left(|u|^{1+\varepsilon}\right)\right|^{p} d x \\
& =(1+\varepsilon)^{p} \int_{\left\{|u|^{1+\varepsilon} \leq M\right\}} a(x)|u|^{p \varepsilon}|\nabla u|^{p} d x \\
& =\frac{(1+\varepsilon)^{p}}{1+\varepsilon p} \int_{\left\{|u|^{1+\varepsilon} \leq M\right\}} a(x)|\nabla u|^{p-2} \nabla u \cdot \nabla\left(|u|^{p \varepsilon} u\right) d x \\
& =C(\varepsilon) \int_{\Omega} a(x)|\nabla u|^{p-2} \nabla u \cdot \nabla \varphi_{M, \varepsilon} d x
\end{aligned}
$$

where $\varphi_{M, \varepsilon}=\min \left(|u|^{p \varepsilon} u^{+}, M^{\frac{1+p \varepsilon}{1+\varepsilon}}\right)+\max \left(|u|^{p \varepsilon} u^{-},-M^{\frac{1+p \varepsilon}{1+\varepsilon}}\right)$.

Part (ii) in Lemma 2.1 yields that $u^{+}, u^{-} \in W_{0}^{1, p}(\Omega, a)$. Through approximation of $u^{+}, u^{-}$ in $W_{0}^{1, p}(\Omega, a)$ by $C_{c}^{\infty}(\Omega)$ functions, we verify that $\varphi_{M, \varepsilon} \in W_{0}^{1, p}(\Omega, a)$.

Testing (1.7) against $\varphi_{M, \varepsilon}$,

$$
\begin{aligned}
\int_{\Omega} a(x)\left|\nabla v_{M, \varepsilon}\right|^{p} d x & =C(\varepsilon) \int_{\Omega} F(x, u) \varphi_{M, \varepsilon} d x \\
& \leq C(\varepsilon) \int_{\Omega}|F(x, u)||u|^{1+\varepsilon p} d x
\end{aligned}
$$


The last inequality and (2.11) yield

$$
\int_{\Omega} a(x)\left|\nabla v_{M, \varepsilon}\right|^{p} d x \leq C(\varepsilon) \int_{\Omega} b(x)|u|^{1+\varepsilon p} d x .
$$

To estimate the right hand side in (2.13) we proceed as follows:

$$
\begin{aligned}
\int_{\Omega} b(x)|u|^{1+\varepsilon p} d x & =\int_{\{|u| \leq 1\}} b(x)|u|^{1+\varepsilon p} d x+\int_{\{|u|>1\}} b(x)|u|^{1+\varepsilon p} d x \\
& \leq \int_{\Omega} b(x) d x+\int_{\{|u|>1\}} b(x)|u|^{1+\varepsilon p}|u|^{p-1} d x \\
& \leq\|b\|_{L^{1}(\Omega)}+\underbrace{\int_{\Omega} b(x)|u|^{p(1+\varepsilon)} d x}_{I}
\end{aligned}
$$

Next we estimate $I$. Let $L>0$ be arbitrary. From (B1) and Hölder's inequality

$$
\begin{aligned}
I & =\int_{\{b \leq L\}} b(x)|u|^{p(1+\varepsilon)} d x+\int_{\{b>L\}} b(x)|u|^{p(1+\varepsilon)} d x \\
& \leq L \int_{\Omega}|u|^{p(1+\varepsilon)} d x+\left(\int_{\Omega} b(x)^{\frac{q}{q-p}} d x\right)^{\frac{q-p}{q}}\left(\int_{\{b>L\}}|u|^{q(1+\varepsilon)} d x\right)^{\frac{p}{q}} \\
& \leq L \int_{\Omega}|u|^{p(1+\varepsilon)} d x+\|b\|_{L^{\frac{q}{q-p}(\Omega)}} \operatorname{meas}\{b>L\}^{\frac{p\left(p_{s}^{*}-q\right)}{q p_{s}^{*}}}\left(\int_{\Omega}|u|^{p_{s}^{*}(1+\varepsilon)} d x\right)^{\frac{p}{p_{s}^{*}}} .
\end{aligned}
$$

Setting

$$
\sigma(L):=\|b\|_{L^{\frac{q}{q-p}(\Omega)}} \operatorname{meas}\{b>L\}^{\frac{p\left(p_{s}^{*}-q\right)}{q p_{s}^{*}}}, \quad c_{0}:=1+\|b\|_{L^{1}(\Omega)}
$$

and putting together (2.13), (2.14) and (2.15), we obtain

$$
\int_{\Omega} a(x)\left|\nabla v_{M, \varepsilon}\right|^{p} d x \leq C(\varepsilon)\left(c_{0}+L \int_{\Omega}|u|^{p(1+\varepsilon)} d x+\sigma(L)\left(\int_{\Omega}|u|^{p_{s}^{*}(1+\varepsilon)} d x\right)^{\frac{p}{p_{s}^{*}}}\right) .
$$

Notice that the right hand side in (2.16) does not depend on $M>0$. Using the Monotone Convergence Theorem we pass to the limit as $M \rightarrow \infty$ to find that

$$
\int_{\Omega} a(x)\left|\nabla\left(|u|^{1+\varepsilon}\right)\right|^{p} d x \leq C(\varepsilon)\left(c_{0}+L \int_{\Omega}|u|^{p(1+\varepsilon)} d x+\sigma(L)\left(\int_{\Omega}|u|^{p_{s}^{*}(1+\varepsilon)} d x\right)^{\frac{p}{p_{s}^{*}}}\right)
$$

which implies that $|u|^{1+\varepsilon} \in W^{1, p}(\Omega, a)$, provided that $|u|^{1+\varepsilon} \in L^{p_{s}^{*}}(\Omega)$.

Using that $p<q<p_{s}^{*}$, set

$$
\kappa_{0}:=\frac{p\left(p_{s}^{*}-q\right)}{p_{s}^{*}(q-p)} \quad \text { and } \quad c_{1}:=\|b\|_{L^{\frac{q}{q-p}}(\Omega)}^{1+\kappa_{0}}>0 .
$$


We estimate

$$
\begin{aligned}
& \sigma(L) \leq\|b\|_{L^{\frac{q}{q-p}}(\Omega)}\left(\int_{\{b>L\}} L^{\frac{q}{q-p}} d x\right)^{\frac{p\left(p_{s}^{*}-q\right)}{q p_{s}^{*}}} L^{-\frac{p\left(p_{s}^{*}-q\right)}{p_{s}^{*}(q-p)}} \\
& \leq\|b\|_{L^{\frac{q}{q-p}(\Omega)}}^{1+\frac{p\left(p_{s}^{*}-q\right)}{p^{*}(q-p)}} L^{-\frac{p(p s}{\left.p_{s}^{*}-q\right)}(q-p)} \\
& =c_{1} L^{-\kappa_{0}} \text {. }
\end{aligned}
$$

Next, fix $\delta>0$ such that $0<\mu_{0}^{p}-\delta^{p}<1$, where $\mu_{0}>0$ is the Sobolev constant in (2.10). Choose $L=L_{\varepsilon}>0$ such that

$$
c_{1} C(\varepsilon) L^{-\kappa_{0}}=\delta^{p}
$$

so that from (2.10), (2.17) and (2.18), we get

$$
\begin{aligned}
\mu_{0}^{p}\left(\int_{\Omega}|u|^{p_{s}^{*}(1+\varepsilon)} d x\right)^{\frac{p}{p_{s}^{*}}} & \leq \int_{\Omega} a(x)\left|\nabla\left(|u|^{1+\varepsilon}\right)\right|^{p} d x \\
& \leq C(\varepsilon)\left(c_{0}+L \int_{\Omega}|u|^{p(1+\varepsilon)} d x\right)+\delta^{p}\left(\int_{\Omega}|u|^{p_{s}^{*}(1+\varepsilon)} d x\right)^{\frac{p}{p_{s}^{*}}} .
\end{aligned}
$$

Since $L=\frac{c_{1}^{\frac{1}{\kappa_{0}}} C^{\frac{1}{\kappa_{0}}}(\varepsilon)}{\delta^{\frac{1}{\kappa_{0}}}},(2.19)$ yields

$$
\left(\mu_{0}^{p}-\delta^{p}\right)\|u\|_{L^{p}(1+\varepsilon)(\Omega)}^{p(1+\varepsilon)} \leq \frac{c_{1}^{\frac{1}{\kappa_{0}}}}{\delta^{\frac{p}{\kappa_{0}}}} C(\varepsilon)^{1+\frac{1}{\kappa_{0}}}\left(\frac{c_{0}}{L}+\|u\|_{L^{p(1+\varepsilon)}(\Omega)}^{p(1+\varepsilon)}\right) .
$$

Using our choice of $L=L_{\varepsilon}$ and setting $\kappa_{1}:=1+\frac{1}{\kappa_{0}}$, there exists $c_{2}>0$ such that

$$
\|u\|_{L^{p^{*}(1+\varepsilon)}(\Omega)} \leq c_{2}^{\frac{1}{p(1+\varepsilon)}} C(\varepsilon)^{\frac{\kappa_{1}}{p(1+\varepsilon)}}\left[\left(\frac{c_{0}}{C(\varepsilon)^{\frac{1}{\kappa_{0}}}}\right)^{\frac{1}{p(1+\varepsilon)}}+\|u\|_{L^{p(1+\varepsilon)}(\Omega)}\right] .
$$

Notice that $c_{0}>1$ and $C(\varepsilon) \geq 1$. Therefore,

$$
\|u\|_{L^{p_{s}^{*}(1+\varepsilon)}(\Omega)} \leq c_{2}^{\frac{1}{p(1+\varepsilon)}} C(\varepsilon)^{\frac{k_{1}}{p(1+\varepsilon)}}\left(c_{0}+\|u\|_{L^{p(1+\varepsilon)}(\Omega)}\right) .
$$

To finish the proof, we use (2.20) and proceed as in the proof of Lemma 3.2 in [9]. For the sake of completeness we include the details.

We run an iterative scheme as follows. Set $\varepsilon_{0}=0$ to find from (2.20) that

$$
\|u\|_{L^{p^{*}}(\Omega)} \leq c_{2}^{\frac{1}{p}}\left(c_{0}+\|u\|_{L^{p}(\Omega)}\right) .
$$


Next, setting $\varepsilon_{1}>0$ such that $p\left(1+\varepsilon_{1}\right)=p_{s}^{*}$ we get from (2.20) and (2.21)

$$
\begin{aligned}
\|u\|_{L^{p}\left(1+\varepsilon_{1}\right)}(\Omega) & \leq c_{2}^{\frac{1}{p\left(1+\varepsilon_{1}\right)}} C\left(\varepsilon_{1}\right)^{\frac{k_{1}}{p\left(1+\varepsilon_{1}\right)}}\left(c_{0}+\|u\|_{L^{p\left(1+\varepsilon_{1}\right)}(\Omega)}\right) \\
& \leq c_{2}^{\frac{1}{p\left(1+\varepsilon_{1}\right)}} C\left(\varepsilon_{1}\right)^{\frac{\kappa_{1}}{p\left(1+\varepsilon_{1}\right)}}\left(c_{0}+c_{2}^{\frac{1}{p}}\left(c_{0}+\|u\|_{L^{p}(\Omega)}\right)\right)
\end{aligned}
$$

and since $C\left(\varepsilon_{0}\right)=1$,

$$
\|u\|_{L^{p_{s}^{*}\left(1+\varepsilon_{1}\right)}(\Omega)} \leq c_{2}^{\frac{1}{p\left(1+\varepsilon_{0}\right)}+\frac{1}{p\left(1+\varepsilon_{1}\right)}} C\left(\varepsilon_{0}\right)^{\frac{\kappa_{1}}{p\left(1+\varepsilon_{0}\right)}} C\left(\varepsilon_{1}\right)^{\frac{\kappa_{1}}{p\left(1+\varepsilon_{1}\right)}}\left(c_{0}\left(1+c_{2}^{-\frac{1}{p}}\right)+\|u\|_{L^{p}(\Omega)}\right) .
$$

For the next iteration we choose $\varepsilon_{2}$ given by $p\left(1+\varepsilon_{2}\right)=p_{s}^{*}\left(1+\varepsilon_{1}\right)$ and from (2.20) and (2.22),

$$
\begin{gathered}
\|u\|_{L^{p_{s}^{*}\left(1+\varepsilon_{2}\right)}(\Omega)} \leq c_{2}^{\frac{1}{p\left(1+\varepsilon_{2}\right)}} C\left(\varepsilon_{2}\right)^{\frac{k_{1}}{p\left(1+\varepsilon_{2}\right)}}\left(c_{0}+\|u\|_{L^{p\left(1+\varepsilon_{2}\right)}(\Omega)}\right) \\
\leq c_{2}^{\frac{1}{p\left(1+\varepsilon_{2}\right)}} C\left(\varepsilon_{2}\right)^{\frac{\kappa_{1}}{p\left(1+\varepsilon_{2}\right)}}\left[c_{0}+c_{2}^{\frac{1}{p\left(1+\varepsilon_{0}\right)}+\frac{1}{p\left(1+\varepsilon_{1}\right)}} C\left(\varepsilon_{0}\right)^{\frac{\kappa_{1}}{p\left(1+\varepsilon_{0}\right)}} C\left(\varepsilon_{1}\right)^{\frac{\kappa_{1}}{p\left(1+\varepsilon_{1}\right)}}\left(c_{0}\left(1+c_{2}^{-\frac{1}{p}}\right)+\|u\|_{L^{p}}\right)\right] \\
\leq c_{2}^{\sum_{j=0}^{2} \frac{1}{p\left(1+\varepsilon_{j}\right)}} \Pi_{j=0}^{2} C\left(\varepsilon_{j}\right)^{\frac{\kappa_{1}}{p\left(1+\varepsilon_{j}\right)}}\left(c_{0}\left(1+c_{2}^{-\frac{1}{p}}+c_{2}^{\sum_{j=0}^{1} \frac{-1}{p\left(1+\varepsilon_{j}\right)}} \Pi_{j=0}^{1} C\left(\varepsilon_{j}\right)^{\frac{-\kappa_{1}}{p\left(1+\varepsilon_{j}\right)}}\right)+\|u\|_{L^{p}}\right)
\end{gathered}
$$

so that

$$
\|u\|_{L^{p_{S}^{*}\left(1+\varepsilon_{2}\right)}} \leq c_{2}^{\sum_{j=0}^{2} \frac{1}{p\left(1+\varepsilon_{j}\right)}} \Pi_{j=0}^{2} C\left(\varepsilon_{j}\right)^{\frac{\kappa_{1}}{p\left(1+\varepsilon_{j}\right)}}\left(c_{0}\left(1+c_{2}^{-\frac{1}{p}}+c_{2}^{\frac{-2}{p}}\right)+\|u\|_{L^{p}}\right) .
$$

We proceed inductively by choosing $\varepsilon_{n}>0$ such that

$$
p\left(1+\varepsilon_{n}\right):=p_{s}^{*}\left(1+\varepsilon_{n-1}\right), \quad \text { i.e. } \quad \frac{1}{1+\varepsilon_{n}}=\left(\frac{p}{p_{s}^{*}}\right)^{n}, \quad n \geq 0
$$

to obtain that

$$
\|u\|_{L^{p_{S}^{*}\left(1+\varepsilon_{n}\right)}} \leq c_{2}^{\frac{1}{p} \sum_{j=0}^{n} \frac{1}{1+\varepsilon_{j}}} \Pi_{j=0}^{n} C\left(\varepsilon_{j}\right)^{\frac{\kappa_{1}}{p\left(1+\varepsilon_{j}\right)}}\left(c_{0} \sum_{m=0}^{n} c_{2}^{\frac{-m}{p}}+\|u\|_{L^{p}}\right) .
$$

Since $p<p_{s}^{*}$,

$$
\sum_{j=0}^{\infty} \frac{1}{1+\varepsilon_{j}}=\sum_{j=0}^{\infty}\left(\frac{p}{p_{s}^{*}}\right)^{j}=\frac{p_{s}^{*}}{p_{s}^{*}-p}<\infty
$$


and

$$
\begin{aligned}
0<\log \left(\Pi_{j=0}^{n} C\left(\varepsilon_{j}\right)^{\frac{\kappa_{1}}{p\left(\varepsilon_{j}+1\right)}}\right) & =\sum_{j=0}^{n} \frac{\kappa_{1}}{\varepsilon_{j}+1} \log \left(\frac{1+\varepsilon_{j}}{\left(1+p \varepsilon_{j}\right)^{\frac{1}{p}}}\right) \\
& \leq \sum_{j=0}^{n} \frac{\kappa_{1}}{\varepsilon_{j}+1} \log \left(\left(1+\varepsilon_{j}\right)^{1-\frac{1}{p}}\right) \\
& =\kappa_{1} \frac{p-1}{p} \log \left(\frac{p_{s}^{*}}{p}\right) \sum_{j=0}^{n} j\left(\frac{p}{p_{s}^{*}}\right)^{j} .
\end{aligned}
$$

Testing the root criterion for convergence of series,

$$
\liminf _{j \rightarrow \infty} \sqrt[j]{j\left(\frac{p}{p_{s}^{*}}\right)^{j}} \leq \frac{p}{p_{s}^{*}}<1
$$

Consequently, in the limit of the iterative argument the sequences

$$
c_{2}^{\frac{1}{p} \sum_{j=1}^{n} \frac{1}{\varepsilon_{j}+1}}, \quad \prod_{j=0}^{n} C\left(p, \varepsilon_{j}\right)^{\frac{\kappa_{1}}{p\left(\varepsilon_{j}+1\right)}}, \quad c_{0} \sum_{m=0}^{n}{c_{2}}^{\frac{-m}{p}} \quad\left(c_{2}>2, \quad p>1\right)
$$

are uniformly bounded. Passing to the limit as $n \rightarrow \infty$ in (2.23) and recalling $c_{0}=$ $1+\|b\|_{L^{1}(\Omega)}$, we obtain

$$
\|u\|_{L^{\infty}(\Omega)} \leq C\left(1+\|b\|_{L^{1}(\Omega)}+\|u\|_{L^{p}(\Omega)}\right) .
$$

The case $p=q$, i.e., $\frac{q}{q-p}=\infty$, follows by choosing any $\tilde{q}$ with $p<\tilde{q}<p_{s}^{*}$ and performing the above estimates, changing $q$ by $\tilde{q}$ where necessary, and using that

$$
\|b\|_{L^{\frac{\tilde{q}}{q-p}(\Omega)}} \leq \operatorname{meas}(\Omega)^{\frac{\tilde{q}}{p}}\|b\|_{L^{\infty}(\Omega)}
$$

and this completes the proof of Proposition 2.1.

Solutions to (1.1) are locally Hölder continuous. This is the content of the next proposition.

Proposition 2.2. Let (A1)-(A2) and (B1) hold. Assume that $F(x, \zeta)$ is a Caratheodory function satisfying (1.6).

Let $D \subset \mathbb{R}^{N}$ be an open set such that $\bar{D} \subset \Omega$. Then, there exist $\alpha \in(0,1)$ and $\kappa>0$, both depending only on $D, a, b, p, q, N$, and there exists $C=C(\Omega, D)>0$ such that for any weak solution $u \in W_{0}^{1, p}(\Omega, a) \cap L^{\infty}(\Omega)$ of (1.1),

(i) $u \in C^{0, \alpha}(\bar{D})$ and

(ii) for any $x, y \in D$,

$$
|u(x)-u(y)| \leq C\left[\kappa+\|u\|_{L^{\infty}(D)}\right]|x-y|^{\alpha} .
$$

Proof. This result is a direct consequence of the inequalities in (1.5) and Theorems 1 and 8 in 21 . 
2.3. Boundary behavior of solutions to (1.1). Next, we review some well-known facts about Fermi coordinates. We refer the reader to [7, 20] for more details on the developments hereby presented.

For any $\rho>0$, set

$$
\Omega_{\rho}:=\{x \in \Omega: 0<\operatorname{dist}(x, \partial \Omega)<\rho\}
$$

and let $\mathrm{n}: \partial \Omega \rightarrow S^{N-1}$ be the inner unit normal vector to $\partial \Omega$.

In what follows, we assume with no loss of generality that the mapping

$$
\mathrm{x}\left(\mathrm{y}, y_{N}\right):=\mathrm{y}+y_{N} \mathrm{n}(\mathrm{y}) \text { for }\left(\mathrm{y}, y_{N}\right) \in \partial \Omega \times\left(0, \rho_{0}\right)
$$

is a smooth diffeomorphism onto $\Omega_{\rho_{0}}($ see $(1.2))$.

Let $\Phi: \mathcal{U} \rightarrow \Phi(\mathcal{U})$ be a local parametrisation of $\partial \Omega$ with $\mathcal{U} \subset \mathbb{R}^{N-1}$ being an open connected set.

For $\left(y, y_{N}\right) \in \mathcal{U} \times\left(0, \rho_{0}\right)$ define

$$
X\left(y, y_{N}\right):=\mathrm{x}\left(\Phi(y), y_{N}\right)=\Phi(y)+y_{N} \mathrm{n}(y),
$$

where, abusing the notation, we have written $\mathrm{n}(y):=\mathrm{n}(\Phi(y))$.

Set $g_{i j}:=\partial_{y_{i}} X \cdot \partial_{y_{j}} X$ for $i, j=1, \ldots, N$. Observe that

$$
\begin{aligned}
\forall i, j & =1, \ldots, N-1: \quad g_{i j}=\partial_{y_{i}} \Phi \cdot \partial_{y_{j}} \Phi+2 y_{N} \partial_{y_{i}} \Phi \cdot \partial_{y_{j}} \mathrm{n}+y_{N}^{2} \partial_{y_{i}} \mathrm{n} \cdot \partial_{y_{j}} \mathrm{n}, \\
\forall i & =1, \ldots, N-1: \quad g_{N i}=g_{i N}=0
\end{aligned}
$$

and $g_{N N}=1$.

Therefore, setting

$$
g:=\left(\begin{array}{cc}
\left(g_{i j}\right)_{N-1 \times N-1} & 0 \\
0 & 1
\end{array}\right) \quad \text { we have } \quad g^{-1}:=\left(\begin{array}{cc}
\left(g^{i j}\right)_{N-1 \times N-1} & 0 \\
0 & 1
\end{array}\right) .
$$

Here $g=\left(g_{i j}\right)_{N \times N}$ is the induced Riemann metric on $X\left(\mathcal{U} \times\left(0, \rho_{0}\right)\right)$ with inverse $g^{-1}=$ $\left(g^{i j}\right)_{N \times N}$. Since $\Omega$ is a smooth domain the matrices $g$ and $g^{-1}$ are smooth in $\partial \Omega \times\left(-\rho_{0}, \rho_{0}\right)$.

Next, we observe that from (A1), (A2) and from the change of variables it follows that

$$
\int_{\Omega_{\rho_{0}}} a(x) d x=\int_{\partial \Omega} \int_{0}^{\rho_{0}} \mathrm{a}\left(y_{N}\right) \sqrt{\operatorname{det} g} d y_{N} d y \leq C_{\Omega} \int_{0}^{\rho_{0}} \mathrm{a}\left(y_{N}\right) d y_{N}
$$

for some constant $C_{\Omega}>0$. We thus conclude that $a \in L^{1}(\Omega)$. Similarly, we can show that $a^{-1} \in L^{s}(\Omega)$. This proves the claim made at the beginning of subsection 2.1 .

In the next lemma we compute $\operatorname{div}\left(a(x)|\nabla w|^{p-2} \nabla w\right)$ in the coordinates $x=X\left(y, y_{N}\right)$ for certain class of functions $w$.

First, for any $\varphi \in W_{l o c}^{1,1}\left(\Omega_{\rho_{0}}\right)$, we write

$$
x=X\left(y, y_{N}\right) \quad \text { and } \quad \varphi(x):=\varphi\left(y, y_{N}\right) \quad \text { for } \quad\left(y, y_{N}\right) \in \mathcal{U} \times\left(0, \rho_{0}\right) .
$$


Notice that in the coordinates $x=X\left(y, y_{N}\right)$,

$$
\nabla_{x} \varphi(x)=\sum_{i, k=1}^{N-1} g^{i k} \partial_{y_{k}} \varphi\left(y, y_{N}\right) \partial_{y_{i}} X+\partial_{y_{N}} \varphi\left(y, y_{N}\right) \mathrm{n}(y) .
$$

Lemma 2.3. Let (A1) and (A2) hold and let $w \in W^{1, p}\left(\Omega_{\rho_{0}}, a\right)$ be such that $w(x):=$ $w(\operatorname{dist}(x, \partial \Omega))$ in $\Omega_{\rho_{0}}$. Then,

(i) for any $\varphi \in C_{c}^{\infty}\left(\Omega_{\rho_{0}}\right)$,

$$
\begin{aligned}
\int_{\Omega_{\rho_{0}}} a(x)|\nabla w|^{p-2} \nabla w \cdot \nabla \varphi & d x \\
& =\int_{\partial \Omega} \int_{0}^{\rho_{0}} \mathrm{a}\left(y_{N}\right)\left|\partial_{y_{N}} w\right|^{p-2} \partial_{y_{N}} w \partial_{y_{N}} \varphi\left(y, y_{N}\right) \sqrt{\operatorname{det} g} d y_{N} d y .
\end{aligned}
$$

(ii) If in addition $\mathrm{a}\left(y_{N}\right)\left|\partial_{y_{N}} w\right|^{p-2} \partial_{y_{N}} w$ is absolutely continuous in $\left(0, \rho_{0}\right)$,

$$
\begin{aligned}
\operatorname{div}\left(a(x)|\nabla w|^{p-2} \nabla w\right)= & \partial_{y_{N}}\left(\mathrm{a}\left(y_{N}\right)\left|\partial_{y_{N}} w\right|^{p-2} \partial_{y_{N}} w\right) \\
& +\partial_{y_{N}} \log (\sqrt{\operatorname{det} g}) \mathrm{a}\left(y_{N}\right)\left|\partial_{y_{N}} w\right|^{p-2} \partial_{y_{N}} w
\end{aligned}
$$

for a.e. $x=X\left(y, y_{N}\right)$ with $\left(y, y_{N}\right) \in \mathcal{U} \times\left(0, \rho_{0}\right)$.

Proof. Identity (2.25) is a direct consequence of (A2) and the change of variables.

Assume now that $\mathrm{a}\left(y_{N}\right)\left|\partial_{y_{N}} w\right|^{p-2} \partial_{y_{N}} w$ is absolutely continuous in $\left(0, \rho_{0}\right)$. Integrating by parts in (2.25),

$$
\operatorname{div}\left(a(x)|\nabla w|^{p-2} \nabla w\right)=\frac{1}{\sqrt{\operatorname{det} g}} \partial_{y_{N}}\left(\sqrt{\operatorname{det} g} \mathrm{a}\left(y_{N}\right)\left|\partial_{y_{N}} w\right|^{p-2} \partial_{y_{N}} w\right) .
$$

Performing the product differentiation in the right-hand side of (2.27), equality (2.26) follows. This completes the proof of the lemma.

Denote

$$
\mathrm{d}(x):=\int_{0}^{\operatorname{dist}(x, \partial \Omega)} \mathrm{a}^{-\frac{1}{p-1}}(\zeta) d \zeta \quad \text { for } \quad x \in \Omega_{\rho_{0}} .
$$

Lemma 2.4. Assume (A1) and (A2). Then there exists a function $\psi \in W^{1, p}(\Omega, a) \cap C(\bar{\Omega})$ with $\left.\psi\right|_{\partial \Omega}=0$ and such that

(i) for every $\varphi \in W_{0}^{1, p}\left(\Omega_{\rho_{0}}, a\right)$ with $\varphi \geq 0$ a.e. in $\Omega_{\rho_{0}}$,

$$
\int_{\Omega_{\rho_{0}}} a(x)|\nabla \psi|^{p-2} \nabla \psi \cdot \nabla \varphi d x \geq \int_{\Omega_{\rho_{0}}} a^{-\frac{1}{p-1}}(x) \varphi d x
$$

and

(ii) there exists a constant $C>0$ such that for every $x \in \Omega_{\rho_{0}}$

$$
\frac{1}{C} \mathrm{~d}(x) \leq \psi(x) \leq C \mathrm{~d}(x) .
$$


Proof. Consider local coordinates $\Phi_{i}: \mathcal{U}_{i} \rightarrow \Phi_{i}(\mathcal{U}) \subset \partial \Omega$ with $\left\{\Phi_{i}\left(\mathcal{U}_{i}\right)\right\}_{i=1}^{m}$ being a finite covering for $\partial \Omega$. Using the geometric notations from Lemma 2.3, set

$$
\Lambda:=\max _{1 \leq i \leq m} \max _{\substack{y \in \overline{\mathcal{U}_{i}}, 0 \leq y_{N} \leq \rho_{0}}}\left|\partial_{y_{N}} \log (\sqrt{\operatorname{det} g})\right|<\infty .
$$

Next, let $A>0$ be a fixed constant such that

$$
A>\int_{0}^{\rho_{0}} e^{\Lambda \tau} \mathrm{a}^{-\frac{1}{p-1}}(\tau) d \tau
$$

and consider the function $\psi:[0, \infty) \rightarrow[0, \infty)$ defined by

$$
\psi\left(y_{N}\right)=\int_{0}^{y_{N}} \frac{1}{e^{\frac{\Lambda \zeta}{p-1}} \mathrm{a}^{\frac{1}{p-1}}(\zeta)}\left[A-\int_{0}^{\zeta} e^{\Lambda \tau} \mathrm{a}^{-\frac{1}{p-1}}(\tau) d \tau\right]^{\frac{1}{p-1}} d \zeta \quad \text { for } \quad y_{N} \in\left[0, \rho_{0}\right] .
$$

Next, we list some properties of the function $\psi$ which are direct consequences of (1.3) and (2.32):

(I) $\psi \in C\left[0, \rho_{0}\right]$ and $\psi(0)=0$;

(II) $\psi$ is differentiable in $\left(0, \rho_{0}\right]$ with

$$
\partial_{y_{N}} \psi\left(y_{N}\right)=e^{-\frac{\Lambda y_{N}}{p-1}} \mathrm{a}^{-\frac{1}{p-1}}\left(y_{N}\right)\left[A-\int_{0}^{y_{N}} e^{\Lambda \tau} \mathrm{a}^{-\frac{1}{p-1}}(\tau) d \tau\right]^{\frac{1}{p-1}} \quad \text { for } \quad y_{N} \in\left(0, \rho_{0}\right)
$$

(III) $\mathrm{a}\left(y_{N}\right)\left|\partial_{y_{N}} \psi\right|^{p-1}$ is absolutely continuous in $\left(0, \rho_{0}\right)$ and

$$
-\partial_{y_{N}}\left(\mathrm{a}\left(y_{N}\right)\left|\partial_{y_{N}} \psi\right|^{p-1}\right)-\Lambda \mathrm{a}\left(y_{N}\right)\left|\partial_{y_{N}} \psi\right|^{p-1}=\mathrm{a}^{\frac{1}{p-1}}\left(y_{N}\right) \quad \text { in } \quad\left(0, \rho_{0}\right) .
$$

Next, observe that a direct calculation yields the estimate

$$
\begin{aligned}
& -\frac{1}{\sqrt{\operatorname{det} g}} \partial_{y_{N}}\left(\sqrt{\operatorname{det} g} \mathrm{a}\left(y_{N}\right)\left|\partial_{y_{N}} \psi\right|^{p-1}\right) \geq-\partial_{y_{N}}\left(\mathrm{a}\left(y_{N}\right)\left|\partial_{y_{N}} \psi\right|^{p-1}\right) \\
& -\left|\partial_{y_{N}} \log (\sqrt{\operatorname{det} g})\right| a\left(y_{N}\right)\left|\partial_{y_{N}} \psi\right|^{p-1} \\
& \geq-\partial_{y_{N}}\left(\mathrm{a}\left(y_{N}\right)\left|\partial_{y_{N}} \psi\right|^{p-1}\right)-\Lambda \mathrm{a}\left(y_{N}\right)\left|\partial_{y_{N}} \psi\right|^{p-1}
\end{aligned}
$$

in $\mathcal{U}_{i} \times\left(0, \rho_{0}\right)$ for every $i=1, \ldots, m$, where the latter inequality in (2.34) follows from the definition of $\Lambda$ in (2.31).

The estimate in (2.34) and the equality in (2.33) imply that

$$
-\frac{1}{\sqrt{\operatorname{det} g}} \partial_{y_{N}}\left(\sqrt{\operatorname{det} g} \mathrm{a}\left(y_{N}\right)\left|\partial_{y_{N}} \psi\right|^{p-1}\right) \geq \mathrm{a}^{-\frac{1}{p-1}}\left(y_{N}\right) \quad \text { in } \quad \mathcal{U}_{i} \times\left(0, \rho_{0}\right)
$$

for every $i=1, \ldots, m$.

Observe also that (II) yields that

$$
\frac{1}{C} \mathrm{a}^{-\frac{1}{p-1}}\left(y_{N}\right) \leq \partial_{y_{N}} \psi\left(y_{N}\right) \leq C \mathrm{a}^{-\frac{1}{p-1}}\left(y_{N}\right) \quad \text { for } \quad y_{N} \in\left(0, \rho_{0}\right),
$$


where

$$
C:=\max \left\{A, e^{\frac{\Lambda \rho_{0}}{p-1}}\left(A-\int_{0}^{\rho_{0}} e^{\Lambda \tau} \mathrm{a}^{-\frac{1}{p-1}}(\tau) d \tau\right)^{-1}\right\}>0 .
$$

Consequently,

$$
\frac{1}{C} \int_{0}^{y_{N}} \mathrm{a}^{-\frac{1}{p-1}}(\zeta) d \zeta \leq \psi\left(y_{N}\right) \leq C \int_{0}^{y_{N}} \mathrm{a}^{-\frac{1}{p-1}}(\zeta) d \zeta
$$

for every $y_{N} \in\left(0, \rho_{0}\right)$.

Set $\psi(x):=\psi(\operatorname{dist}(x, \partial \Omega))$ for $x \in \bar{\Omega}_{\rho_{0}}$ (see (1.2) $)$ and extend it continuously as $\psi(x):=$ $\psi\left(\rho_{0}\right)$ in $\Omega-\Omega_{\rho_{0}}$. From (I) and (II), it is direct to verify that $\psi \in W^{1, p}(\Omega, a) \cap C(\bar{\Omega}), \psi>0$ in $\Omega$ and $\left.\psi\right|_{\partial \Omega}=0$.

Estimate (2.35) and part (ii) from Lemma 2.3, imply that

$$
-\operatorname{div}\left(a(x)|\nabla \psi|^{p-2} \nabla \psi\right) \geq a^{-\frac{1}{p-1}}(x) \quad \text { in } \quad \Omega_{\rho_{0}} .
$$

To prove (i) we use (2.37) and Lemma 2.3 to find, after integrating by parts, that for every $\varphi \in C_{c}^{\infty}\left(\Omega_{\rho_{0}}\right)$ with $\varphi \geq 0$ in $\Omega_{\rho_{0}}$,

$$
\int_{\Omega_{\rho_{0}}} a(x)|\nabla \psi|^{p-2} \nabla \psi \cdot \nabla \varphi d x \geq \int_{\Omega_{\rho_{0}}} a^{-\frac{1}{p-1}}(x) \varphi d x .
$$

The Dominated convergence Theorem implies that (2.38) holds true for every $\varphi \in W_{0}^{1, p}\left(\Omega_{\rho_{0}}, a\right)$.

Finally, the proof of the inequalities (2.30) in (ii) readily follows from the definition of $\psi(x)$, the definition of $\mathrm{d}(x)$ in $(2.28)$ and the inequalities in (2.36). This completes the proof of the lemma.

Our final result in this section deals with boundary behavior of solutions of (1.1) under an assumption related to (1.6).

Proposition 2.3. Assume (A1) and (A2) and let $\rho \in\left(0, \rho_{0}\right]$ be fixed. Let $F: \Omega \times \mathbb{R} \rightarrow \mathbb{R}$ be a Caratheodory function such that

$$
\sup _{x \in \Omega_{\rho}, \zeta \in \mathbb{R}} a^{\frac{1}{p-1}}(x)|F(x, \zeta)|<+\infty .
$$

There exists a constant $\tilde{C}>0$ such that for every solution $u \in W_{0}^{1, p}(\Omega, a) \cap L^{\infty}(\Omega)$ of (1.1),

$$
\left\|\mathrm{d}^{-1} u\right\|_{L^{\infty}\left(\Omega_{\rho}\right)} \leq \tilde{C}\left(1+\|u\|_{L^{\infty}(\Omega)}\right) .
$$

Proof. Let $u \in W_{0}^{1, p}(\Omega, a) \cap L^{\infty}(\Omega)$ be a solution of (1.1). From (2.39) there exists $\mathcal{K}>0$ such that

$$
|F(x, u(x))| \leq \mathcal{K} a^{-\frac{1}{p-1}}(x) \quad \text { a.e. } x \in \Omega_{\rho} .
$$

Let $\psi \in W_{0}^{1, p}(\Omega, a) \cap C(\bar{\Omega})$ be the function predicted in Lemma 2.4. We compare the solution $u$ of (1.1) with an appropriate multiple of $\psi$ in $\Omega_{\rho}$. 
Fix $M>0$ such that

$$
M^{p-1}>\mathcal{K} \quad \text { and } \quad M \int_{0}^{\rho} \mathrm{a}^{-\frac{1}{p-1}}(\zeta) d \zeta \geq C,
$$

where $C$ is the constant in (2.30).

Set $\bar{u}(x)=M\left(1+\|u\|_{L^{\infty}(\Omega)}\right) \psi(x)$ for $x \in \Omega$. We claim that $(u-\bar{u})^{+} \in W_{0}^{1, p}\left(\Omega_{\rho}, a\right)$. Let us assume for the moment that this claim has been proven. We would then proceed as follows.

Extend the functions in $W_{0}^{1, p}\left(\Omega_{\rho}, a\right)$ to $\Omega_{\rho_{0}}$ and the functions in $W_{0}^{1, p}\left(\Omega_{\rho_{0}}, a\right)$ to $\Omega$ by defining them as zero in $\Omega_{\rho_{0}}-\Omega_{\rho}$ and $\Omega-\Omega_{\rho_{0}}$, respectively. We obtain the embeddings:

$$
W_{0}^{1, p}\left(\Omega_{\rho}, a\right) \hookrightarrow W_{0}^{1, p}\left(\Omega_{\rho_{0}}, a\right) \hookrightarrow W_{0}^{1, p}(\Omega, a) .
$$

Next, choosing $\varphi=(u-\bar{u})^{+}$in (1.7) and (2.29) and using the monotonicity of the vector function

$$
\mathbb{R}^{N} \ni z \mapsto|z|^{p-2} z
$$

together with (2.41), we get

$$
\begin{aligned}
0 & \geq \int_{\Omega_{\rho} \cap\{u \geq \bar{u}\}} a(x)\left(|\nabla \bar{u}|^{p-2} \nabla \bar{u}-|\nabla u|^{p-2} \nabla u\right) \cdot \nabla(u-\bar{u}) d x \\
& =\int_{\Omega_{\rho}} a(x)\left(|\nabla \bar{u}|^{p-2} \nabla \bar{u}-|\nabla u|^{p-2} \nabla u\right) \cdot \nabla(u-\bar{u})^{+} d x \\
& \geq \int_{\Omega_{\rho}}\left(M^{p-1} a^{-\frac{1}{p-1}}(x)-F(x, u)\right)(u-\bar{u})^{+} d x \\
& \geq \int_{\Omega_{\rho}}\left(M^{p-1}-\mathcal{K}\right) a^{-\frac{1}{p-1}}(x)(u-\bar{u})^{+} d x .
\end{aligned}
$$

From (2.42),

$$
\int_{\Omega_{\rho}}\left(M^{p-1}-\mathcal{K}\right) a^{-\frac{1}{p-1}}(x)(u-\bar{u})^{+} d x \geq 0
$$

and consequently $(u-\bar{u})^{+}=0$, i.e. $u \leq \bar{u}$ in $\Omega_{\rho}$.

Proceeding in a similar fashion for $-u$, we obtain that

$$
|u(x)| \leq \bar{u}(x)=M\left(1+\|u\|_{L^{\infty}(\Omega)}\right) \psi(x) \quad \text { for } \quad x \in \Omega_{\rho} .
$$

Inequality (2.40) follows from (2.30) and (2.43). This would complete the proof of the proposition.

Next, we prove the claim. First notice that $u-\bar{u} \in W_{0}^{1, p}(\Omega, a)$ and Lemma 2.1 yields that $(u-\bar{u})^{+} \in W_{0}^{1, p}(\Omega, a)$.

Fix $\sigma \in(0, \rho)$ and let $\chi: \Omega \rightarrow[0, \infty)$ be a non-negative smooth function such that

$$
\chi(x)=\left\{\begin{array}{lll}
0, & \text { for } & x \in \Omega-\Omega_{\sigma} \\
1, & \text { for } & x \in \Omega_{\frac{\sigma}{2}}
\end{array}\right.
$$


Since

$$
(u-\bar{u})^{+}:=\chi(u-\bar{u})^{+}+(1-\chi)(u-\bar{u})^{+} \quad \text { in } \Omega,
$$

it suffices to prove that $\chi(u-\bar{u})^{+}$and $(1-\chi)(u-\bar{u})^{+}$belong to $W_{0}^{1, p}\left(\Omega_{\rho}, a\right)$. We prove these two statements as follows.

First, approximating $(u-\bar{u})^{+}$in $W_{0}^{1, p}(\Omega, a)$ with functions in $C_{c}^{\infty}(\Omega)$ yields $\chi(u-\bar{u})^{+} \in$ $W_{0}^{1, p}\left(\Omega_{\rho}, a\right)$.

On the other hand, from Proposition [2.2, $u \in C(\Omega)$ and thus $(u-\bar{u})^{+} \in C\left(\bar{\Omega}_{\rho}-\partial \Omega\right)$. Using (2.28), (2.30) and (2.42), we find that $\bar{u} \geq u$ on $\{\operatorname{dist}(\cdot, \partial \Omega)=\rho\}$ and hence

$$
(u-\bar{u})^{+}=0 \quad \text { on } \quad\{\operatorname{dist}(\cdot, \partial \Omega)=\rho\} .
$$

Consequently, $(1-\chi)(u-\bar{u})^{+} \in W^{1, p}\left(\Omega_{\rho}, a\right) \cap C\left(\bar{\Omega}_{\rho}\right)$ and from (2.44),

$$
\left.(1-\chi)(u-\bar{u})^{+}\right|_{\partial \Omega_{\rho}}=0 .
$$

Lemma 2.2 implies that $(1-\chi)(u-\bar{u})^{+} \in W_{0}^{1, p}\left(\Omega_{\rho}, a\right)$. This proves the claim and also completes the proof of the proposition.

\section{Proof of Theorem 1.1}

In this part we follow the scheme from Chapter 5 in [10] to prove Theorem 1.1. Also, we make use of the notations and conventions introduced in Sections 1 and 2.

Assume hypotheses (A1)-(A2) and (B1)-(B2). Using (B2), let $\rho_{1} \in\left(0, \rho_{0}\right)$ be such that

$$
\forall x \in \Omega_{\rho_{1}}: \quad c_{1} a^{-\frac{1}{p-1}}(x) \leq b(x) \leq c_{2} a^{-\frac{1}{p-1}}(x),
$$

where $\Omega_{\rho_{1}}$ is as in (2.24).

Consider the space $C(\bar{\Omega})$ endowed with the norm $\|\cdot\|_{L^{\infty}(\Omega)}$ and its subspace

$$
C_{0}(\bar{\Omega}):=\{w \in C(\bar{\Omega}): w=0 \quad \text { on } \quad \partial \Omega\} .
$$

Next, consider the space

$$
Y_{a}:=\left\{\tilde{b} \in L^{\frac{q}{q-p}}(\Omega): a^{\frac{1}{p-1}}|\tilde{b}| \in L^{\infty}\left(\Omega_{\rho_{1}}\right)\right\}
$$

where $q \in\left[p, p_{s}^{*}\right)$ is described in hypothesis (B1) and $Y_{a}$ is endowed with the norm

$$
\|\tilde{b}\|_{Y_{a}}:=\|\tilde{b}\|_{L^{\frac{q}{q-p}(\Omega)}}+\left\|a^{\frac{1}{p-1}} \tilde{b}\right\|_{L^{\infty}\left(\Omega_{\rho_{1}}\right)}
$$

Recall also that $\frac{q}{q-p}:=\infty$ if $q=p$.

The following lemma will help us to set up the functional analytic settings to o carry out the proof of Theorem 1.1. 
Lemma 3.1. Let $r \in\left[\frac{p_{s}^{*}}{p_{s}^{*}-1}, \infty\right]$ and $\tilde{b} \in L^{r}(\Omega)$. The $B V P$

$$
-\operatorname{div}\left(a(x)|\nabla w|^{p-2} \nabla w\right)=\tilde{b}(x) \quad \text { in } \quad \Omega, \quad w=0 \quad \text { on } \quad \partial \Omega,
$$

has a unique solution $w \in W_{0}^{1, p}(\Omega, a)$, which satisfies the estimate

$$
\|w\|_{W_{0}^{1, p}(\Omega, a)} \leq C\|\tilde{b}\|_{L^{r}(\Omega)}^{\frac{1}{p-1}}
$$

for some constant $C>0$, depending only on $p, s, r, \Omega$ and the function $a$.

If in addition $\tilde{b} \in Y_{a}$, then the solution $w$ of (3.2) belongs also to $C_{0}(\bar{\Omega})$, it is locally Hölder continuous in $\Omega$ and satisfies the estimate

$$
\|w\|_{L^{\infty}(\Omega)}+\left\|\mathrm{d}^{-1} w\right\|_{L^{\infty}\left(\Omega_{\rho_{1}}\right)} \leq C\left(1+\|\tilde{b}\|_{L^{\frac{q}{q-p}}(\Omega)}+\|\tilde{b}\|_{L^{\frac{q}{q-p}}(\Omega)}^{\frac{1}{p-1}}\right)
$$

for some constant $C>0$ depending only on $p, q, s, \Omega$ and the function $a$.

Proof. Given $r \in\left[\frac{p_{s}^{*}}{p_{s}^{*}-1}, \infty\right]$ and $\tilde{b} \in L^{r}(\Omega)$, the existence of a unique solution $w \in W_{0}^{1, p}(\Omega, a)$ of (3.2), follows readily by a minimization procedure and a weak lower semicontinuity argument or by the use of theory of monotone operators.

Set $\frac{r}{r-1}:=1$, if $r=\infty$. The weak form of (3.2) (see (1.7)), the fact that $\frac{r}{r-1} \in\left[1, p_{s}^{*}\right]$ together with Hölder's inequality and the Sobolev embedding in (2.10), imply that

$$
\|w\|_{W_{0}^{1, p}(\Omega, a)}^{p}=\int_{\Omega} \tilde{b} w d x \leq\|\tilde{b}\|_{L^{r}(\Omega)}\|w\|_{L^{\frac{r}{r-1}(\Omega)}} \leq \tilde{C}\|\tilde{b}\|_{L^{r}(\Omega)}\|w\|_{W_{0}^{1, p}(\Omega, a)}
$$

for some constant $\tilde{C}>0$ depending only on $p, s, r, \Omega$ and $a$. The estimate in (3.3) follows taking $C=\tilde{C}^{\frac{1}{p-1}}$.

Assume now that $\tilde{b} \in Y_{a}$. Propositions 2.1 and 2.2 imply, respectively, that $w \in L^{\infty}(\Omega)$ and $w$ is locally Hölder continuous in $\Omega$. Also, the estimate (2.12) in Proposition 2.1 yields that

$$
\|w\|_{L^{\infty}(\Omega)} \leq C\left(1+\|\tilde{b}\|_{L^{1}(\Omega)}+\|w\|_{L^{p}(\Omega)}\right)
$$

and from the Friedrich's inequality, the estimate in (3.3) with $r=\frac{q}{q-p}$ and Hölder's inequality we find that

$$
\|w\|_{L^{\infty}(\Omega)} \leq C\left(1+\|\tilde{b}\|_{L^{\frac{q}{q-p}(\Omega)}}+\|\tilde{b}\|_{L^{\frac{q}{q-p}(\Omega)}}^{\frac{1}{p-1}}\right) .
$$

Next, we apply Proposition 2.3 , with $\rho=\rho_{1}$, to find that $w \in C_{0}(\bar{\Omega})$. The estimate in (3.4) follows from the estimate (3.5) and the estimate (2.40) in Proposition 2.3, again with $\rho=\rho_{1}$. This completes the proof of the lemma.

Using Proposition [3.1, let $\mathcal{L}: L^{\frac{p_{s}^{*}}{p_{s}^{*}-1}}(\Omega) \rightarrow W_{0}^{1, p}(\Omega, a)$ denote the resolvent operator of (3.2) and observe that $\mathcal{L}: Y_{a} \rightarrow C_{0}(\bar{\Omega})$ is well defined.

Lemma 3.2. The operator $\mathcal{L}: Y_{a} \rightarrow C_{0}(\bar{\Omega})$ is compact and continuous. 
Proof. Step 1: compactness. Assume $\left\{\tilde{b}_{n}\right\}_{n} \subset Y_{a}$ is bounded. Thus, there exists $\mathcal{K}>0$ such that

$$
\forall n \in \mathbb{N}: \quad\left\|\tilde{b}_{n}\right\|_{L^{\frac{q}{q-p}}(\Omega)}+\left\|a^{\frac{1}{p-1}} \tilde{b}_{n}\right\|_{L^{\infty}\left(\Omega_{\rho_{1}}\right)} \leq \mathcal{K} .
$$

Noticing that $L^{\frac{q}{q-p}}(\Omega) \subset L^{\frac{p_{s}^{*}}{p_{s}^{*}-1}}(\Omega)$ and using the reflexivity and separability of the space $L^{\frac{p_{s}^{*}}{p_{s}^{*}-1}}(\Omega)$ we may assume, passing to a subsequence if necessary, the existence of $\tilde{b}_{0} \in$ $L^{\frac{p_{*}^{*}}{p_{s}^{*}-1}}(\Omega)$ such that for every $\varphi \in W_{0}^{1, p}(\Omega, a)$,

$$
\lim _{n \rightarrow \infty} \int_{\Omega} \tilde{b}_{n}(x) \varphi d x=\int_{\Omega} \tilde{b}_{0}(x) \varphi d x .
$$

Next, for every $n \in \mathbb{N} \cup\{0\}$, set

$$
w_{n}:=\mathcal{L}\left(\tilde{b}_{n}\right)
$$

and observe from (3.3), (3.6) and (3.8) that $\left\{w_{n}\right\}_{n \in \mathbb{N} \cup\{0\}}$ is bounded in $W_{0}^{1, p}(\Omega, a)$.

Proceeding as in the Proof of Theorem 1.1 in [1] (p. 1163-1164) using the embeddings in (2.9) and the reflexivity and separability of the space $W_{0}^{1, p}(\Omega, a)$, we find $\tilde{w}_{0} \in W_{0}^{1, p}(\Omega, a)$ such that up to a subsequence,

$$
\lim _{n \rightarrow \infty} \int_{\Omega} a(x)\left|\nabla w_{n}\right|^{p-2} \nabla w_{n} \cdot \nabla \varphi d x=\int_{\Omega} a(x)\left|\nabla \tilde{w}_{0}\right|^{p-2} \nabla \tilde{w}_{0} \cdot \nabla \varphi d x, \quad \forall \varphi \in W_{0}^{1, p}(\Omega, a) .
$$

We can assume also that $w_{n}(x) \rightarrow \tilde{w}_{0}(x)$ a.e. $x \in \Omega$.

Putting together (3.7), (3.8) and (3.9), we obtain that

$$
\int_{\Omega}\left(a(x)\left|\nabla \tilde{w}_{0}\right|^{p-2} \nabla \tilde{w}_{0} \cdot \nabla \varphi-\tilde{b}_{0}(x) \cdot \varphi\right) d x=0, \quad \forall \varphi \in W_{0}^{1, p}(\Omega, a) .
$$

From (3.10) and Lemma 3.1, $\tilde{w}_{0}=\mathcal{L}\left(\tilde{b}_{0}\right)=w_{0} \in W_{0}^{1, p}(\Omega, a)$.

The definition of $w_{n}$ in (3.8), together with the estimates in (3.4) and (3.6) yield that the sequence $\left\{w_{n}\right\}_{n}$ is bounded in $L^{\infty}(\Omega)$.

Next, we prove the existence of a subsequence of $\left\{w_{n}\right\}_{n}$, say $\left\{w_{n_{k}}\right\}_{k}$, such that

$$
w_{n_{k}}=\mathcal{L}\left(\tilde{b}_{n_{k}}\right) \rightarrow w_{0}=\mathcal{L}\left(\tilde{b}_{0}\right) \text { strongly in } L^{\infty}(\Omega) .
$$

Proposition 2.2 applied to each domain in the sequence of open sets

$$
D_{k}:=\left\{x \in \Omega: \operatorname{dist}(x, \partial \Omega)>\frac{1}{k}\right\} \quad \text { for } \quad k \in \mathbb{N},
$$

and performing a domain approximation procedure (see the proof of Theorem 1.1 in [1] p. 1164-1166) and a diagonal argument, we pass to a subsequence, denoted again by $\left\{w_{n}\right\}_{n}$, such that $w_{n} \rightarrow w_{0}$ uniformly over compact sets of $\Omega$.

Using again the estimates in (3.4) and (3.6), we find a constant $c>0$ such that for every $n \in \mathbb{N}$ and every $x \in \Omega_{\rho_{1}}$,

$$
\left.\left|w_{n}(x)\right|\right) \leq c \mathrm{~d}(x)
$$


Since $w_{n}(x) \rightarrow w_{0}(x)$ for a.e. $x \in \Omega$, we also find that for a.e. $x \in \Omega_{\rho_{1}}$,

$$
\left|w_{0}(x)\right| \leq c \mathrm{~d}(x) \text {. }
$$

We conclude that

$$
\left\|w_{n}\right\|_{L^{\infty}\left(\Omega_{\rho}\right)}+\left\|w_{0}\right\|_{L^{\infty}\left(\Omega_{\rho}\right)} \rightarrow 0, \quad \text { as } \quad \rho \rightarrow 0
$$

uniformly in $n \in \mathbb{N}$, where $\Omega_{\rho}$ is defined in (2.24).

The fact that $w_{n} \rightarrow w_{0}$ uniformly over compact subsets of $\Omega$ and (3.12) imply that $w_{n} \rightarrow w_{0}$ strongly in $L^{\infty}(\Omega)$. This completes the proof of the Step 1 .

Step 2: continuity. We proceed as in Step 1 with only slight changes. The details are left to the reader. This completes the proof of the lemma.

Next, let $F=F(x, \zeta)$ be a Caratheodory function and let $\underline{u}, \bar{U} \in W^{1, p}(\Omega, a) \cap C(\bar{\Omega})$ be a subsolution and a supersolution to (1.1), respectively. Assume further that $\underline{u} \leq \bar{U}$ in $\bar{\Omega}$.

Consider the truncated BVP

$$
-\operatorname{div}\left(a(x)|\nabla u|^{p-2} \nabla u\right)=\tilde{F}(x, u) \quad \text { in } \quad \Omega, \quad u=0 \quad \text { on } \quad \partial \Omega,
$$

where

$$
\widetilde{F}(x, \zeta):=\left\{\begin{array}{lc}
F(x, \zeta), & \underline{u}(x) \leq \zeta \leq \bar{U}(x) \\
F(x, \underline{u}(x)), & \zeta \leq \underline{u}(x) \\
F(x, \bar{U}(x)), & \bar{U}(x) \leq \zeta
\end{array} \quad \text { for } \quad x \in \Omega, \quad \zeta \in \mathbb{R} .\right.
$$

Observe that $\tilde{F}$ is also a Caratheodory function.

Lemma 3.3. Let $u \in W^{1, p}(\Omega, a) \cap C_{0}(\bar{\Omega})$. If $u$ is a solution of (3.13), then $u$ is also $a$ solution of (1.1) with $\underline{u} \leq u \leq \bar{U}$ in $\Omega$.

Proof. Let $u \in W^{1, p}(\Omega, a) \cap C_{0}(\bar{\Omega})$ be a solution of (3.13). Since $\underline{u} \leq 0$ on $\partial \Omega,(\underline{u}-u)^{+}$ belongs to $W^{1, p}(\Omega, a) \cap C_{0}(\bar{\Omega})$. Lemma 2.1 implies that $(\underline{u}-u)^{+} \in W_{0}^{1, p}(\Omega, a)$.

From (1.7), with $\tilde{F}(x, \zeta)$ instead of $F(x, \zeta)$, and (1.8),

$$
\begin{aligned}
0 & \leq \int_{\Omega} a(x)\left(|\nabla \underline{u}|^{p-2} \nabla \underline{u}-|\nabla u|^{p-2} \nabla u\right) \cdot \nabla(\underline{u}-u)^{+} d x \\
& \leq \int_{\{\underline{u} \geq u\}}(F(x, \underline{u})-\widetilde{F}(x, u))(\underline{u}-u)^{+} d x=0
\end{aligned}
$$

from where we obtain $\underline{u} \leq u$ in a.e. $\Omega$. Proceeding in similar fashion we can verify that $\bar{U} \geq u$. This proves the lemma.

Let $F$ satisfy (1.6). Set

$$
M:=\max \left(\|\underline{u}\|_{L^{\infty}(\Omega)},\|\bar{U}\|_{L^{\infty}(\Omega)}\right)+1
$$

so that there exists $C_{M}>0$ such that for a.e. $x \in \Omega$ and every $\zeta \in \mathbb{R}$,

$$
|\tilde{F}(x, \zeta)| \leq C_{M} b(x) .
$$


Thanks to (3.1) and (3.14) $\tilde{F}(x, \zeta)$ satisfies (2.39) and for any $u \in C_{0}(\bar{\Omega}), \tilde{F}(\cdot, u(\cdot)) \in Y_{a}$.

From Lemma 3.1, the operator

$$
T: C_{0}(\bar{\Omega}) \rightarrow C_{0}(\bar{\Omega}), \quad T(u):=\mathcal{L}(\tilde{F}(\cdot, u(\cdot))), \quad u \in C_{0}(\bar{\Omega})
$$

is well defined.

Proof of Theorem 1.1. Step 1: compactness and continuity of $T$. The compactness of $T$ is a direct consequence of (3.14) and Lemma 3.2.

To prove the continuity of $T$ we proceed as follows. Let $\left\{u_{n}\right\}_{n \in \mathbb{N} \cup\{0\}} \subset C_{0}(\bar{\Omega})$ such that $u_{n} \rightarrow u_{0}$ uniformly in $\Omega$. In particular,

$$
\forall x \in \Omega: \quad u_{n}(x) \rightarrow u_{0}(x), \quad \text { as } \quad n \rightarrow \infty .
$$

Since $\tilde{F}$ is a Caratheodory function,

$$
\forall x \in \Omega: \quad \tilde{F}\left(x, u_{n}(x)\right) \rightarrow \tilde{F}\left(x, u_{0}(x)\right), \quad \text { as } \quad n \rightarrow \infty .
$$

For every $n \in \mathbb{N} \cup\{0\}$, set

$$
\tilde{b}_{n}:=\tilde{F}\left(\cdot, u_{n}(\cdot)\right) \quad \text { and } \quad w_{n}:=\mathcal{L}\left(\tilde{b}_{n}\right)=T\left(u_{n}\right) .
$$

We remark that $\left\{\tilde{b}_{n}\right\}_{n \in \mathbb{N}} \subset Y_{a}$, but it may not converge in $Y_{a}$ and hence the continuity of $T$ does not follow directly from the continuity of $\mathcal{L}$.

We overcome this difficulty as follows. Due to (3.14), (3.6) is satisfied. This fact, together with (3.15) and the Dominated convergence Theorem imply that (3.7) holds true.

Proceeding in the same fashion as in Step 1 in the proof of Lemma 3.2, we find that passing to a subsequence if necessary, (3.11) holds also true as well, i.e.

$$
T\left(u_{n}\right) \rightarrow T\left(u_{0}\right) \quad \text { strongly in } L^{\infty}(\Omega) .
$$

This proves the continuity of $T$ and completes the proof of Step 1.

Step 2: existence. Let $u \in C_{0}(\bar{\Omega})$ and set $w:=\tilde{T}(u)$, i.e., $w=\mathcal{L}(\tilde{F}(\cdot, u(\cdot)))$. Estimate (3.4) with $\tilde{b}=\tilde{F}(\cdot, u(\cdot))$ and inequality (3.14) imply the existence of a constant $\hat{C}=$ $\hat{C}(\underline{u}, \bar{U}, p, q, a, b)>0$ such that

$$
\|w\|_{L^{\infty}(\Omega)} \leq \hat{C}\left(1+\|b\|_{L^{\frac{q}{q-p}(\Omega)}}+\|b\|_{L^{\frac{q}{q-p}}(\Omega)}^{\frac{1}{p-1}}\right) .
$$

Setting

$$
R_{0}:=\hat{C}\left(1+\|b\|_{L^{\frac{q}{q-p}}(\Omega)}+\|b\|_{L^{\frac{q}{q-p}}(\Omega)}^{\frac{1}{p-1}}\right)
$$

we conclude that for every $u \in C_{0}(\bar{\Omega})$,

$$
\|T(u)\|_{L^{\infty}(\Omega)} \leq R_{0} .
$$

Fix $R>R_{0}$ and let $B_{R}$ denote the open ball in $C_{0}(\bar{\Omega})$ of radius $R>0$ and centered at the origin and notice that $\tilde{T}\left(B_{R}\right) \subset B_{R}$. 
Schauder's Fixed Point Theorem yields the existence of $u \in B_{R}$ such that $u=T(u)$. Lemma 3.1 implies that $u \in W_{0}^{1, p}(\Omega, a) \cap C(\bar{\Omega})$ and solves (3.13).

Finally, Lemma 3.3 implies that $u$ also solves (1.1) and this completes the proof of Theorem 1.1.

\section{Proof of theorem 1.2 .}

In this final section we prove Theorem 1.2 Besides (A1)-(A2) and (B1)-(B2), we assume also (f1)-(f3). We adopt the notations and conventions from the previous sections.

Proposition 4.1. For every $\lambda>0$, there exist $\bar{U}_{\lambda} \in W^{1, p}(\Omega, a) \cap C_{0}(\bar{\Omega})$ a supersolution to (1.9) with $\bar{U}_{\lambda}>0$ in $\Omega$.

Although the proof of Proposition 4.1 is rather standard, we include it here for the sake of completeness.

Proof. Let $\lambda>0$ be arbitrary. Set $\bar{U}=M \psi$, where $\psi$ is the solution to (3.2) with $\tilde{b}=b$ and $M>0$ is a parameter to be chosen later. Since $b(x) \geq 0$, from Harnack's inequality (see Theorem 1.9, [9] $), \bar{U}$ is strictly positive inside $\Omega$ and from Lemma [3.1, $\bar{U} \in W^{1, p}(\Omega, a) \cap$ $C_{0}(\bar{\Omega})$.

Consider the function

$$
\hat{f}(\zeta):=\max _{\mathrm{z} \in[0, \zeta]} f(\mathrm{z})
$$

which is monotone increasing, $\hat{f} \geq f$ and from $(f 1)$,

$$
\limsup _{\zeta \rightarrow \infty} \frac{\hat{f}(\zeta)}{\zeta^{p-1}}=0 .
$$

Fix $\varepsilon=\varepsilon(\lambda)>0$ such that

$$
\varepsilon\|\psi\|_{L^{\infty}(\Omega)}^{p-1} \leq \frac{1}{\lambda}
$$

Using (4.1) we get the existence of $\zeta_{\lambda}>1$ such that for every $\zeta \geq \zeta_{\lambda}$,

$$
0 \leq \hat{f}(\zeta) \leq \varepsilon \zeta^{p-1}
$$

Choose $M=M(\lambda)$ satisfying

$$
M\|\psi\|_{L^{\infty}} \geq \zeta_{\lambda}
$$

so that

$$
\hat{f}\left(M\|\psi\|_{L^{\infty}(\Omega)}\right) \leq \varepsilon M^{p-1}\|\psi\|_{L^{\infty}(\Omega)}^{p-1}
$$

and from (4.2),

$$
\lambda \hat{f}\left(M\|\psi\|_{L^{\infty}(\Omega)}\right) \leq M^{p-1} .
$$


Then, formally, we compute

$$
\begin{aligned}
\lambda b(x) \hat{f}\left(M\|\psi\|_{L^{\infty}(\Omega)}\right) & \leq M^{p-1} b(x) \\
& =-\operatorname{div}\left(a(x)|\nabla \bar{U}|^{p-2} \nabla \bar{U}\right)
\end{aligned}
$$

so that $\bar{U}$ is a supersolution to (1.9), proving the proposition.

Proposition 4.2. Let $\Omega$ be a smooth bounded domain such that $\partial \Omega$ has non-negative mean curvature. There exists $\lambda_{*}>0$ such that for every $\lambda \geq \lambda_{*}$, there exists $\underline{u}_{\lambda} \in W^{1, p}(\Omega, a) \cap$ $C_{0}(\bar{\Omega})$ a subsolution to (1.9) with $\underline{u}_{\lambda}>0$ in $\Omega$.

Proof. First, we describe the general strategy. Let $r \in(0, p-1)$ be as in (f2). Thanks to (f1), (f2) and (f3), we can also fix a monotone increasing function $\tilde{f} \in C[0, \infty)$ such that

$$
\tilde{f} \leq f, \quad-\infty<\tilde{f}(0)<f(0) \leq 0
$$

and, moreover, satisfying that

$$
\mu:=\lim _{\zeta \rightarrow \infty} \frac{\tilde{f}(\zeta)}{\zeta^{r}} \in(0, \infty) .
$$

Thus, it suffices to find a subsolution for the BVP

$$
-\operatorname{div}\left(a(x)|\nabla u|^{p-2} \nabla u\right)=\lambda b(x) \tilde{f}(u) \quad \text { in } \quad \Omega, \quad u=0 \quad \text { on } \quad \partial \Omega .
$$

To do so, we proceed in a series of steps. In what follows, we fix $\beta$ and $\sigma$ such that

$$
\frac{1}{p-1}<\sigma<\frac{1}{p-1-r} \text { and } 1<\beta<1+\frac{1}{p-1}
$$

Step 1: profile near $\partial \Omega$. Let $\rho_{1} \in\left(0, \rho_{0}\right)$ be as in (3.1). Since $\partial \Omega$ has non-negative mean curvature, we may also assume that $\rho_{1}$ is such that

$$
\Lambda:=\min _{\substack{\mathrm{y} \in \partial \Omega, 0 \leq y_{N} \leq \rho_{1}}} \partial_{y_{N}} \log (\sqrt{\operatorname{det} g}) \geq 0 .
$$

Consider the decreasing function $A:\left[\frac{\rho_{1}}{2}, \rho_{1}\right) \rightarrow(0, \infty)$ defined by

$$
A(\rho):=\beta^{p-1}\left[\int_{0}^{\rho} \mathrm{a}^{-\frac{1}{p-1}}(\tau) d \tau\right]^{-(p-1)} .
$$

We claim that there exists $\hat{\lambda}_{1}>0$ such that for every $\lambda \geq \hat{\lambda}_{1}$, there exists $\rho=\rho(\lambda) \in$ $\left(\frac{\rho_{1}}{2}, \rho_{1}\right)$ satisfying that

$$
e^{\Lambda \rho} A(\rho)=\lambda^{1-\sigma(p-1-r)} \int_{\rho}^{\rho_{1}} e^{-\Lambda \tau} \mathrm{a}^{-\frac{1}{p-1}}(\tau) d \tau
$$

To prove the claim, consider the positive function

$$
\left[\frac{\rho_{1}}{2}, \rho_{1}\right) \ni \rho \mapsto \mathrm{t}(\rho):=\frac{\mathrm{e}^{\Lambda \rho} A(\rho)}{\int_{\rho}^{\rho_{1}} e^{\Lambda \tau} \mathrm{a}^{-\frac{1}{p-1}}(\tau) d \tau}
$$


so that $\mathrm{t} \in C\left[\frac{\rho_{1}}{2}, \rho_{1}\right)$ and $\mathrm{t}(\rho) \rightarrow+\infty$ as $\rho \rightarrow \rho_{1}$. Let $\hat{\rho} \in\left[\frac{\rho_{1}}{2}, \rho_{1}\right)$ be such that $\mathrm{t}(\hat{\rho}):=$ $\min \mathrm{t}(\rho)$ and notice that $\mathrm{t}(\hat{\rho})>0$.

$\rho \in\left[\frac{\rho_{1}}{2}, \rho_{1}\right)$

Setting

$$
\hat{\lambda}_{1}:=\mathrm{t}(\hat{\rho})^{\frac{1}{1-\sigma(p-1-r)}},
$$

we observe that the claim follows by a direct application of the Intermediate Value Theorem.

Next, we consider the function $\rho:\left[\hat{\lambda}_{1}, \infty\right) \rightarrow\left[\frac{\rho_{1}}{2}, \rho_{1}\right)$, where $\rho=\rho(\lambda)$ solves (4.9).

Next, let $\lambda \geq \hat{\lambda}_{1}$. Write $\rho=\rho(\lambda)$ and consider the function $\phi:\left[\rho, \rho_{1}\right] \rightarrow(0, \infty)$ defined by

$$
\phi\left(y_{N}\right):=1+\int_{\rho}^{y_{N}} e^{-\frac{\Lambda \zeta}{p-1}} \mathrm{a}^{-\frac{1}{p-1}}(\zeta)\left[e^{\Lambda \rho} A(\rho)-\lambda^{1-\sigma(p-1-r)} \int_{\rho}^{\zeta} e^{\Lambda \tau} \mathrm{a}^{-\frac{1}{p-1}}(\tau) d \tau\right]^{\frac{1}{p-1}} d \zeta
$$

Using (1.3) we verify that $\phi$ is well defined, differentiable and $a\left(y_{N}\right)|\phi|^{p-1}$ is absolutely continuous in $\left(\rho, \rho_{1}\right)$.

On the other hand, (4.9) yields that for any $y_{N} \in\left(\rho, \rho_{1}\right)$,

$$
e^{\Lambda \rho} A(\rho)>\lambda^{1-\sigma(p-1-r)} \int_{\rho}^{y_{N}} e^{\Lambda \tau} \mathrm{a}^{-\frac{1}{p-1}}(\tau) d \tau
$$

Thus, from (4.10) and (4.11),

$$
\phi(\rho)=1 \quad \text { and } \quad \mathrm{a}\left(y_{N}\right)\left|\partial_{y_{N}} \phi\right|^{p-2} \partial_{y_{N}} \phi>0 \text { in }\left(\rho, \rho_{1}\right) .
$$

Directly from (4.10),

$$
-\partial_{y_{N}}\left(e^{\Lambda y_{N}} \mathrm{a}\left(y_{N}\right)\left|\partial_{y_{N}} \phi\right|^{p-2} \partial_{y_{N}} \phi\right)=\lambda^{1-\sigma(p-1-r)} e^{\Lambda y_{N}} \mathrm{a}^{-\frac{1}{p-1}}\left(y_{N}\right) \quad \text { in } \quad\left(\rho, \rho_{1}\right)
$$

with the boundary conditions

$$
\mathrm{a}(\rho)\left|\partial_{y_{N}} \phi(\rho)\right|^{p-2} \partial_{y_{N}} \phi(\rho)=A(\rho), \quad \mathrm{a}\left(\rho_{1}\right)\left|\partial_{y_{N}} \phi\left(\rho_{1}\right)\right|^{p-2} \partial_{y_{N}} \phi\left(\rho_{1}\right)=0 .
$$

Step 2: candidate for a subsolution of (4.5). Consider the decreasing function $B$ : $\left[\frac{\rho_{1}}{2}, \rho_{1}\right) \rightarrow(0, \infty)$ defined by

$$
B(\rho):=\left[\int_{0}^{\rho} \mathrm{a}^{-\frac{1}{p-1}}(\zeta) d \zeta\right]^{-\beta}
$$

and recall that for $\lambda \in\left[\hat{\lambda}_{1}, \infty\right)$, we write $\rho=\rho(\lambda)$.

Next, consider the function $\mathrm{v}:[0, \infty) \rightarrow \mathbb{R}$ defined by

$$
\mathrm{v}\left(y_{N}\right)=\left\{\begin{array}{cc}
B(\rho)\left(\int_{0}^{y_{N}} \mathrm{a}^{-\frac{1}{p-1}}(\zeta) d \zeta\right)^{\beta}, & 0 \leq y_{N} \leq \rho, \\
\phi\left(y_{N}\right), & \rho<y_{N}<\rho_{1}, \\
\phi\left(\rho_{1}\right), & y_{N} \geq \rho_{1} .
\end{array}\right.
$$

From (4.10), (4.14) and (4.15), $\mathrm{v} \in C[0, \infty) \cap L^{\infty}(0, \infty)$ and $\mathrm{a}\left(y_{N}\right)\left|\partial_{y_{N}} \mathrm{v}\right|^{p-} \partial_{y_{N}} \mathrm{v} \in$ $L^{1}(0, \infty)$ and it is absolutely continuous in $(0, \infty)$. 
Consider the Fermi coordinates (see Section 2.3)

$$
\left(\mathrm{y}, y_{N}\right) \in \partial \Omega \times\left[0, \rho_{1}\right] \mapsto x=\mathrm{y}+y_{N} \mathrm{n}(\mathrm{y}) \in \bar{\Omega}_{\rho_{1}}
$$

with induced metric denoted by $g$ and associated Jacobian determinant $\sqrt{\operatorname{det} g}$. Recall that $y_{N}=\operatorname{dist}(x, \partial \Omega)$.

Set

$$
v(x)=\left\{\begin{array}{cc}
\mathrm{v}(\operatorname{dist}(x, \partial \Omega)), & x \in \bar{\Omega}_{\rho_{1}}, \\
\mathrm{v}\left(\rho_{1}\right), & x \in \Omega-\bar{\Omega}_{\rho_{1}} .
\end{array}\right.
$$

The properties of $\mathrm{v}$ imply that $v \in W^{1, p}(\Omega, a) \cap C_{0}(\bar{\Omega})$.

Omitting the explicit dependence on $\lambda$, set $\underline{u}=\lambda^{\sigma} v$ in $\Omega$. Abusing the notation, write $\underline{u}(x)=\underline{u}\left(y_{N}\right)$ in $\bar{\Omega}_{\rho_{1}}$. Next, we compute $a(x)|\nabla \underline{u}|^{p-2} \nabla \underline{u}$ in Fermi coordinates using Lemma 2.3. From (4.8), (4.10) and (4.15),

$$
\begin{aligned}
\mathrm{a}\left(y_{N}\right)\left|\partial_{y_{N}} \underline{u}\right|^{p-2} \partial_{y_{N} \underline{u}} & =\lambda^{\sigma(p-1)} \mathrm{a}\left(y_{N}\right)\left|\partial_{y_{N}} v\right|^{p-2} \partial_{y_{N}} v \\
& =\left\{\begin{aligned}
\lambda^{\sigma(p-1)} B(\rho)^{p-1} \beta^{p-1}\left[\int_{0}^{y_{N}} \mathrm{a}^{-\frac{1}{p-1}}(\zeta) d \zeta\right]^{(\beta-1)(p-1)}, & 0<y_{N} \leq \rho, \\
\lambda^{\sigma(p-1)} \mathrm{a}\left(y_{N}\right)\left|\partial_{y_{N}} \phi\right|^{p-2} \partial_{y_{N}} \phi, & \rho<y_{N}<\rho_{1} .
\end{aligned}\right.
\end{aligned}
$$

From (4.13), (4.14) and (4.17), we conclude that $\mathrm{a}\left(y_{N}\right)\left|\partial_{y_{N}} \underline{u}\right|^{p-2} \partial_{y_{N}} \underline{u}$ is absolutely continuous in $\left(0, \rho_{1}\right)$.

Step 3: our candidate is indeed a subsolution of (4.5). We now claim that there exists $\lambda_{*} \geq \hat{\lambda}_{1}$ such that for any $\lambda \geq \lambda_{*}, \underline{u}$ is a subsolution to (4.5). To prove this claim, we proceed as follows.

Let $\varphi \in C_{c}^{\infty}(\Omega)$, with $\varphi \geq 0$ in $\Omega$, be arbitrary. Abusing the notation, write in coordinates $\varphi(x)=\varphi\left(\mathrm{y}, y_{N}\right)$ in $\Omega_{\rho_{1}}$.

Using Lemma 2.3 and integrating by parts, we find that

$$
\begin{aligned}
\int_{\Omega} a(x)|\nabla \underline{u}|^{p-2} \nabla \underline{u} \cdot \nabla \varphi d x= & \int_{\partial \Omega} \int_{0}^{\rho_{1}} \mathrm{a}\left(y_{N}\right)\left|\partial_{y_{N}} \underline{u}\right|^{p-2} \partial_{y_{N}} \underline{u} \partial_{y_{N}} \varphi \sqrt{\operatorname{det} g} d y_{N} d \mathrm{y} \\
= & \underbrace{\int_{\partial \Omega} \mathrm{a}\left(y_{N}\right)\left[\partial_{y_{N}} \underline{u}\left(y_{N}\right)\right]^{p-1} \varphi\left(\mathrm{y}, y_{N}\right) \sqrt{\operatorname{det} g\left(\mathrm{y}, y_{N}\right)} \mid \begin{array}{l}
y_{N}=\rho_{1} \\
y_{N}=0
\end{array} d \mathrm{y}}_{I} \\
& \underbrace{-\int_{\partial \Omega} \int_{0}^{\rho_{1}} \partial_{y_{N}}\left(\sqrt{\operatorname{det} g} \mathrm{a}\left(y_{N}\right)\left|\partial_{y_{N}} \underline{u}\right|^{p-2} \partial_{y_{N}} \underline{u}\right) \varphi d y_{n} d \mathrm{y}}_{I I} .
\end{aligned}
$$

From (4.14) and since $\varphi \in C_{c}^{\infty}(\Omega), I=0$ and therefore

$$
\int_{\Omega} a(x)|\nabla \underline{u}|^{p-2} \nabla \underline{u} \cdot \nabla \varphi d x=I I .
$$


Next, we estimate $I I$. Observe that

$$
\begin{aligned}
I I & =\int_{\partial \Omega} \int_{0}^{\rho_{1}}-\frac{1}{\sqrt{\operatorname{det} g}} \partial_{y_{N}}\left(\sqrt{\operatorname{det} g} \mathrm{a}\left(y_{N}\right)\left|\partial_{y_{N}} \underline{u}\right|^{p-2} \partial_{y_{N}} \underline{u}\right) \varphi \sqrt{\operatorname{det} g} d y_{N} d \mathrm{y} \\
& =\underbrace{\int_{\partial \Omega} \int_{0}^{\rho} \cdots \sqrt{\operatorname{det} g} d y_{N} d \mathrm{y}}_{I I_{1}}+\underbrace{\int_{\partial \Omega} \int_{\rho}^{\rho_{1}} \cdots \sqrt{\operatorname{det} g} d y_{N} d y}_{I I_{2}},
\end{aligned}
$$

where we remark again that $\rho=\rho(\lambda)$ and $\rho(\lambda) \in\left[\frac{\rho_{1}}{2}, \rho_{1}\right)$.

We estimate first $I I_{1}$. The choices of $\sigma$ and $\beta$ in (4.6), allow us to fix $\lambda_{*} \geq \hat{\lambda}_{1}$ such that

$$
\underbrace{\lambda_{*}^{1-\sigma(p-1)} c_{2}(-\tilde{f}(0))}_{>0} \leq \beta^{p-1} B\left(\rho_{1}\right)^{(p-1)}(\beta-1)(p-1)\left[\int_{0}^{\rho_{1}} \mathrm{a}^{-\frac{1}{p-1}}(\zeta) d \zeta\right]^{(\beta-1)(p-1)-1},
$$

where $c_{2}>0$ is described in (3.1).

From (4.7), (4.19) and the fact that $B(\rho)$ is decreasing, we obtain for every $\lambda \geq \lambda_{*}$ and for every $y_{N} \in(0, \rho(\lambda))$ that

$$
\begin{aligned}
-\lambda c_{2} \mathrm{a}^{-\frac{1}{p-1}}\left(y_{N}\right) \tilde{f}(0) & {\left[\int_{0}^{y_{N}} \mathrm{a}^{-\frac{1}{p-1}}(\zeta) d \zeta\right]^{(\beta-1)(p-1)-1} \mathrm{a}^{-\frac{1}{p-1}}\left(y_{N}\right) } \\
\leq \lambda^{\sigma(p-1)} \beta^{p-1} B(\rho)^{(p-1)}((\beta-1)(p-1) & \left.+\left[\int_{0}^{y_{N}} \mathrm{a}^{-\frac{1}{p-1}}(\zeta) d \zeta\right]^{(\beta-1)(p-1)} \Lambda\right)
\end{aligned}
$$

Consequently, (4.7) and (4.20) yield that

$$
\begin{aligned}
-\lambda c_{2} \mathrm{a}^{-\frac{1}{p-1}}\left(y_{N}\right) \tilde{f}(0) \leq \lambda^{\sigma(p-1)} \beta^{p-1} B(\rho)^{(p-1)}\left((\beta-1)(p-1)\left[\int_{0}^{y_{N}} \mathrm{a}^{-\frac{1}{p-1}}(\zeta) d \zeta\right]^{(\beta-1)(p-1)-1} \mathrm{a}^{-\frac{1}{p-1}}\left(y_{N}\right)\right. & \left.+\left[\int_{0}^{y_{N}} \mathrm{a}^{-\frac{1}{p-1}}(\zeta) d \zeta\right]^{(\beta-1)(p-1)} \partial_{\left.y_{N} \log (\sqrt{\operatorname{det} g})\right)}\right) . \\
= & \frac{\lambda^{\sigma(p-1)} \beta^{p-1} B(\rho)^{(p-1)}}{\sqrt{\operatorname{det} g}} \partial_{y_{N}}\left(\sqrt{\operatorname{det} g}\left[\int_{0}^{y_{N}} \mathrm{a}^{-\frac{1}{p-1}}(\zeta) d \zeta\right]^{(\beta-1)(p-1)}\right)
\end{aligned}
$$

From the previous inequality and (4.17) we deduce that

$$
-\frac{1}{\sqrt{\operatorname{det} g}} \partial_{y_{N}}\left(\sqrt{\operatorname{det} g} \mathrm{a}\left(y_{N}\right)\left|\partial_{y_{N}} \underline{u}\right|^{p-2} \partial_{y_{N}} \underline{u}\right) \leq \lambda c_{2} \mathrm{a}^{-\frac{1}{p-1}}\left(y_{N}\right) \tilde{f}(0) \quad \text { in } \quad \partial \Omega \times(0, \rho) \text {. }
$$


Using (4.21) and the fact that $\varphi \geq 0$,

$$
\begin{aligned}
I I_{1} & =\int_{\partial \Omega} \int_{0}^{\rho}-\frac{1}{\sqrt{\operatorname{det} g}} \partial_{y_{N}}\left(\sqrt{\operatorname{det} g} \mathrm{a}\left(y_{N}\right)\left|\partial_{y_{N}} \underline{u}\right|^{p-2} \partial_{y_{N}} \underline{u}\right) \varphi \sqrt{\operatorname{det} g} d y_{N} d \mathrm{y} \\
& \leq \int_{\partial \Omega} \int_{0}^{\rho} \lambda c_{2} \mathrm{a}^{-\frac{1}{p-1}}\left(y_{N}\right) \tilde{f}(0) \varphi \sqrt{\operatorname{det} g} d y_{N} d \mathrm{y} .
\end{aligned}
$$

Pulling back the change of variables,

$$
I I_{1} \leq \int_{\Omega_{\rho}} \lambda c_{2} a^{-\frac{1}{p-1}}(x) \tilde{f}(0) \varphi d x \leq 0 .
$$

It then follows from (3.1) and the fact that $\tilde{f}$ is non-decreasing that

$$
I I_{1} \leq \int_{\Omega_{\rho}} \lambda b(x) \tilde{f}(\underline{u}) \varphi d x .
$$

Next, we estimate the integral $I I_{2}$. Since $\underline{u}\left(y_{N}\right)=\lambda^{\sigma} v\left(y_{N}\right)=\lambda^{\sigma} \phi\left(y_{N}\right)$ for $y_{N} \in\left(\rho, \rho_{1}\right)$, we have

$$
I I_{2}=\lambda^{\sigma(p-1)} \int_{\partial \Omega} \int_{\rho}^{\rho_{1}}-\frac{1}{\sqrt{\operatorname{det} g}} \partial_{y_{N}}\left(\sqrt{\operatorname{det} g} \mathrm{a}\left(y_{N}\right)\left|\partial_{y_{N}} \phi\right|^{p-2} \partial_{y_{N}} \phi\right) \varphi \sqrt{\operatorname{det} g} d y_{N} d y .
$$

From (4.12), the function $\phi\left(y_{N}\right), y_{N} \in\left[\rho, \rho_{1}\right)$, is strictly increasing, bounded and $\phi(\rho)=1$. This fact and (4.13) imply that

$$
\begin{aligned}
-\frac{1}{\sqrt{\operatorname{det} g}} \partial_{y_{N}}\left(\sqrt{\operatorname{det} g} \mathrm{a}\left(y_{N}\right)\left|\partial_{y_{N}} \phi\right|^{p-2} \partial_{y_{N}} \phi\right) & \leq \lambda^{1-\sigma(p-1-r)} \mathrm{a}^{-\frac{1}{p-1}}\left(y_{N}\right) \\
& \leq \lambda^{1-\sigma(p-1-r)} \mathrm{a}^{-\frac{1}{p-1}}\left(y_{N}\right) \phi^{r}
\end{aligned}
$$

in $\left(\rho, \rho_{1}\right)$. Therefore,

$$
I I_{2} \leq \lambda^{1-\sigma(p-1)+\sigma r} \int_{\partial \Omega} \int_{\rho}^{\rho_{1}} \mathrm{a}^{-\frac{1}{p-1}}\left(y_{N}\right) \phi^{r} \varphi \sqrt{\operatorname{det} g} d y_{N} d \mathrm{y} .
$$

Pulling back the change of variables and using (3.1) and the definition of $v$ in (4.16), we find that

$$
\begin{aligned}
I I_{2} & \leq \frac{\lambda^{1-\sigma(p-1)}}{c_{1}} \int_{\Omega_{\rho_{1}}-\bar{\Omega}_{\rho}} b(x) \lambda^{\sigma r} \phi^{r} \varphi d x \\
& \leq \frac{\lambda^{1-\sigma(p-1)}}{c_{1}} \int_{\Omega-\bar{\Omega}_{\rho}} b(x) \lambda^{\sigma r} v^{r} \varphi d x .
\end{aligned}
$$

We finish estimating the integral $I I_{2}$ as follows. Fix $\varepsilon \in(0, \mu)$, where $\mu>0$ is as in (4.4). Taking $\lambda_{*}$ larger if necessary, we may assume that

$$
\frac{\lambda_{*}^{-\sigma(p-1)}}{c_{1}(\mu-\varepsilon)} \leq 1
$$

Moreover, thanks to (4.4) and the fact that $v \geq 1$ in $\Omega_{\rho_{1}}-\bar{\Omega}_{\rho}$, we may also assume that for every $\lambda \geq \lambda_{*}$,

$$
(\mu-\varepsilon) \lambda^{\sigma r} v^{r} \leq \tilde{f}\left(\lambda^{\sigma} v\right) \quad \text { in } \quad \Omega_{\rho_{1}}-\bar{\Omega}_{\rho} .
$$


From (4.24), (4.25) and (4.26),

$$
\begin{aligned}
I I_{2} & \leq \int_{\Omega-\bar{\Omega}_{\rho}} \lambda^{1-\sigma(p-1)} b(x) \lambda^{\sigma r} v^{r} \varphi d x \\
& \leq \frac{\lambda^{1-\sigma(p-1)}}{c_{1}(\mu-\varepsilon)} \int_{\Omega-\bar{\Omega}_{\rho}} b(x) \tilde{f}\left(\lambda^{\sigma} v\right) \varphi d x \\
& \leq \lambda \int_{\Omega-\bar{\Omega}_{\rho}} b(x) \tilde{f}(\underline{u}) \varphi d x
\end{aligned}
$$

for every $\lambda \geq \lambda_{*}$.

Finally, putting together (4.23) and (4.27), for every $\lambda \geq \lambda_{*}$,

$$
\int_{\Omega} a(x)|\nabla \underline{u}|^{p-2} \nabla \underline{u} \cdot \nabla \varphi d x \leq \int_{\Omega} \lambda b(x) \tilde{f}(\underline{u}) \varphi d x .
$$

Since $\varphi \in C_{c}^{\infty}(\Omega)$ with $\varphi \geq 0$ in $\Omega$ is arbitrary, we conclude that for any $\lambda \geq \lambda_{*}, \underline{u} \in$ $W^{1, p}(\Omega, a) \cap C_{0}(\bar{\Omega})$ is a subsolution of (1.9) . This complete the proof of the proposition.

Proposition 4.3. Under the same assumptions as in Proposition 4.2, there exists $\lambda_{0}>0$ such that for every $\lambda \geq \lambda_{0}$, there exist $\underline{u}_{\lambda}, \bar{U}_{\lambda}$ a positive subsolution and a positive super solution to (1.9) in $\bar{\Omega}$ with $\underline{u}_{\lambda} \leq \bar{U}_{\lambda}$ in $\bar{\Omega}$.

Proof. We track down the proofs of Propositions 4.1 and 4.2, Let $\lambda_{*}>0$ be as in the proof of Proposition 4.2 and let $M=M(\lambda), \lambda \geq \lambda_{*}$, be as in (4.3). Let also $\underline{u}_{\lambda}, \bar{U}_{\lambda}$ be as in Propositions 4.1 and 4.2, respectively.

Let $\varphi \in W_{0}^{1, p}(\Omega, a) \cap C(\bar{\Omega})$ with $\varphi \geq 0$ be arbitrary. From the definition of the function $v$ in (4.16) and the estimates in (4.18), (4.22) and (4.24), we find that

$$
\begin{aligned}
\int_{\Omega} a(x)\left|\nabla \underline{u}_{\lambda}\right|^{p-2} \nabla \underline{u}_{\lambda} \cdot \nabla \varphi d x \leq \int_{\Omega_{\rho}} \lambda c_{2} a^{-\frac{1}{p-1}}(x) \tilde{f}(0) \varphi d x \\
\quad+\int_{\Omega-\Omega_{\rho}} \frac{\lambda^{1-\sigma(p-1)+\sigma r}}{c_{1}} b(x) v^{r} \varphi d x
\end{aligned}
$$

Since $\tilde{f}(0) \leq 0$, we conclude that

$$
\int_{\Omega} a(x)\left|\nabla \underline{u}_{\lambda}\right|^{p-2} \nabla \underline{u}_{\lambda} \cdot \nabla \varphi d x \leq \int_{\Omega} \frac{\lambda^{1-\sigma(p-1)+\sigma r}}{c_{1}} b(x) v^{r} \varphi d x
$$

for every $\varphi \in C_{c}^{\infty}(\Omega)$ with $\varphi \geq 0$ in $\Omega$.

Therefore,

$$
\begin{aligned}
0 & \leq \int_{\Omega} a(x)\left(\left|\nabla \underline{u}_{\lambda}\right|^{p-2} \nabla \underline{u}_{\lambda}-\left|\nabla \bar{U}_{\lambda}\right|^{p-2} \nabla \bar{U}_{\lambda}\right) \cdot \nabla\left(\underline{u}_{\lambda}-\bar{U}_{\lambda}\right)^{+} d x \\
& \leq \int_{\left\{\underline{u}_{\lambda} \geq \bar{U}_{\lambda}\right\}} b(x)\left(\frac{\lambda^{1-\sigma(p-1-r)}}{c_{1}} v^{r}(x)-M^{p-1}\right)\left(\underline{u}_{\lambda}-\bar{U}_{\lambda}\right)^{+} d x .
\end{aligned}
$$


Next, observe from (4.12) that the function $\mathrm{v}\left(y_{N}\right), y_{N} \in[0, \infty)$, defined in (4.15) is strictly increasing and bounded with $\|\mathrm{v}\|_{L^{\infty}[0, \infty)}=\phi\left(\rho_{1}\right)$. The latter fact together with the definition of $v$ in (4.16) imply that $\|v\|_{L^{\infty}(\Omega)}=\phi\left(\rho_{1}\right)$.

We may take $\lambda_{0} \geq \lambda_{*}$ and for any $\lambda \geq \lambda_{0}$ we may choose $M=M(\lambda)$ larger if necessary, satisfying (4.3) and

$$
c_{1} \lambda^{\sigma(p-1-r)-1} M^{p-1}(\lambda) \geq \phi^{r}\left(\rho_{1}\right)=\|v\|_{L^{\infty}(\Omega)}^{r} .
$$

Consequently,

$$
\int_{\left\{\underline{u}_{\lambda} \geq \bar{U}_{\lambda}\right\}} b(x)\left(\frac{\lambda^{1-\sigma(p-1-r)}}{c_{1}} v^{r}(x)-M^{p-1}\right)\left(\underline{u}_{\lambda}-\bar{U}_{\lambda}\right)^{+} d x \leq 0
$$

and thus $\left(\underline{u}_{\lambda}-\bar{U}_{\lambda}\right)^{+}=0$, i.e., $\underline{u}_{\lambda} \leq \bar{U}_{\lambda}$ in $\Omega$. This completes the proof of the proposition.

Proof Theorem 1.2. Let $\lambda_{0}$ be as in Proposition 4.3 and let $\lambda \geq \lambda_{0}$ be arbitrary, but fixed. Omitting the dependence on $\lambda$, let $\bar{U}, \underline{u}$ be the ordered subsolution and supersolution to (1.9), respectively, predicted in Propositions 4.1, 4.2 and 4.3.

Set $\zeta_{0}:=\max \left(\|\underline{u}\|_{L^{\infty}(\Omega)},\|\bar{U}\|_{L^{\infty}(\Omega)}\right)+1$ and consider the function

$$
F(x, \zeta):=\left\{\begin{array}{cc}
\lambda b(x) f(\zeta), & |\zeta| \leq \zeta_{0} \\
\lambda b(x) f\left(\zeta_{0}\right), & |\zeta|>\zeta_{0}
\end{array}\right.
$$

With this definition of $F$, it is clear that (1.6) is satisfied and $\underline{u}, \bar{U}$ are a sub- and a supersolution of (1.1), respectively.

A direct application of Theorem 1.1 yields the existence of $u \in W_{0}^{1, p}(\Omega) \cap C_{0}(\bar{\Omega})$, a solution of (1.1), such that $0<\underline{u} \leq u \leq \bar{U}$ in $\Omega$.

From (4.28), the function $u$ solves (1.9) and this completes the proof of the theorem.

\section{REFERENCES}

[1] O. Agudelo, P. DrÁBeK. Anisotropic semipositone quasilinear problems. J. Math. Anal. Appl. 452 (2017), no. 2, 1145-1167.

[2] I. Ali, A. Castro, R. Shivaji. Uniqueness and stability of nonnegative solutions for semipositone problems in a ball. Proc. Amer. Math. Soc. 113 (1993), 775-782

[3] A. Ambrosetti, D. Arcoya, and B. Buffoni. Positive solutions for some semi-positone problems via bifurcation theory. Differential Integral Equations 7 (1994), no. 3-4, 655-663.

[4] K. J. Brown, A. Castro, R. Shivaji. Nonexistence of radially symmetric nonnegative solutions for a class of semi-positone problems. Differential Integral Equations 2 (1989), 541-545.

[5] A. Castro, M. Chhetri, R. Shivaji, Nonlinear eigenvalue problems with semipositone structure, Electronic J. Diff. Eqns. Conf. 05 (2000), No. 5, 33-49.

[6] E. Dancer,J. Shi. Uniqueness and nonexistence of positive solutions to semipositone problems. Bull. London Math. Soc. 38 (2006), no. 6, 1033-1044.

[7] M. Do Carmo. Riemannian geometry. Translated from the second Portuguese edition by Francis Flaherty. Mathematics: Theory and Applications. Birkhäuser Boston, Inc., Boston, MA, 1992. xiv+300 pp. ISBN: 0-8176-3490-8.

[8] P. Drábek, L. Sankar. Singular quasilinear elliptic problems on unbounded domains. Nonlinear Anal. 109 (2014), 148-155. 
[9] P. Drábek, A. Kufner, F. Nicolosi. Quasilinear elliptic equations with degenerations and singularities. de Gruyter Series in Nonlinear Analysis and Applications, 5. Walter de Gruyter Co, Berlin, 1997.

[10] P. Drábek, J. Milota. Methods of nonlinear analysis. Applications to differential equations. Second edition. Birkhäuser Advanced Texts: Basler Lehrbücher. Birkhäuser/Springer Basel AG, Basel, 2013. doi: 10.1007/978-3-0348-0387-8.

[11] L.C. Evans. Partial differential equations. Graduate Studies in Mathematics, 19. American Mathematical Society, Providence, RI, 1998. xviii+662 pp. ISBN: 0-8218-0772-2

[12] L.C. Evans, R.F. Gariepy Measure theory and fine properties of functions. Studies in Advanced Mathematics. CRC Press, Boca Raton, FL, 1992. viii+268 pp. ISBN: 0-8493-7157-0

[13] F. Gazzola, Nonlinearity in oscillating bridges. Electron. J. Differential Equations (2013), No. 211, 47 pp.

[14] M. Gromov. Sign and geometric meaning of curvature. Rend. Sem. Mat. Fis. Milano 61 (1991), 9-123 (1994).

[15] D. Gilbarg, N. Trudinger. Elliptic Partial Differential Equations of Second Order, second edition. Springer-Verlag, Berlin (1983).

[16] D. D. HAi, R. Shivaji. Existence and uniqueness for a class of quasilinear elliptic boundary value problems. J. Differential Equations 193 (2003), 500-510.

[17] S. Herrón, E. LoperA. Non-existence of positive radial solution for semipositone weighted pLaplacian problem. Electron. J. Differential Equations 2015, No. 130, 9 pp.

[18] U. Kaufmann, H. Ramos Quoirin. Positive solutions of indefinite semipositone problems via subsuper solutions. Differential Integral Equations 31 (2018), no. 7-8, 497-506.

[19] E. K. Lee, R. Shivaj,, J. Ye. Positive solutions for elliptic equations involving nonlinearities with falling zeroes. Appl. Math. Lett., In press, Available online 17 October (2008).

[20] F.K. Manasse, C.W. Misner. Fermi normal coordinates and some basic concepts in differential geometry. J. Mathematical Phys. 41963 735-745.

[21] J. SERrin. Local behavior of solutions of quasi-linear equations. Acta Math. 111 1964 247-302.

[22] M. Struwe. Variational Methods. Applications to Nonlinear Partial Differential Equations and Hamiltonian Systems. Series: Ergebnisse der Mathematik und ihrer Grenzgebiete. 3. Folge / A Series of Modern Surveys in Mathematics, Vol. 34 4th ed. 2008

[23] P. Tolksdorf. Regularity for a more general class of quasilinear elliptic equations. J. Differential Equations 51 (1984), no. 1, 126-150.

[24] J. L. VAzquez. A strong maximum principle for some quasilinear elliptic equations. Appl. Math. Optim. 12 (1984), 191-202.

O. Agudelo - NTiS, Department of Mathematics, Západočeská Univerzita v Plzni, Plzeñ, Czech Republic.

Email address: oiagudel@ntis.zcu.cz

P. Drábek - ZCU, Department of Mathematics, ZÁpadočeská Univerzita v Plzni, Plzeň, Czech Republic.

Email address: pdrabek@kma.zcu.cz 